\title{
GAMA/H-ATLAS: THE DUST OPACITY-STELLAR MASS SURFACE DENSITY RELATION FOR SPIRAL GALAXIES
}

\author{
M. W. Grootes ${ }^{1}$, R. J. Tuffs ${ }^{1}$, C. C. Popescu ${ }^{2}$, B. Pastrav ${ }^{2}$, E. Andrae ${ }^{1}$, M. Gunawardhana ${ }^{3}$, L. S. Kelvin ${ }^{4,5}$, J. Liske ${ }^{6}$, \\ M. Seibert ${ }^{7}$, E. N. TAYlor ${ }^{3}$, Alister W. Graham ${ }^{8}$, M. Baes ${ }^{9}$, I. K. Baldry ${ }^{10}$, N. Bourne ${ }^{11}$, S. Brough $^{12}$, A. Cooray $^{13}$, \\ A. Dariush ${ }^{14}$, G. De Zotti ${ }^{15,16}$, S. P. Driver ${ }^{4,5}$, L. Dunne ${ }^{17}$, H. GomeZ ${ }^{18}$, A. M. Hopkins ${ }^{12}$, R. Hopwood ${ }^{14,19}$, M. JARVis $^{20,21}$, \\ J. Loveday ${ }^{22}$, S. Maddox ${ }^{17}$, B. F. Madore ${ }^{7}$, M. J. Michałowski ${ }^{23}$, P. Norberg ${ }^{24}$, H. R. Parkinson ${ }^{23}$, M. Prescott $^{10}$, \\ A. S. G. Robotham ${ }^{4,5}$, D. J. B. Smith ${ }^{20}$, D. Thomas ${ }^{25}$, and E. Valiante ${ }^{18}$ \\ ${ }^{1}$ Max-Planck-Institut für Kernphysik, Saupfercheckweg 1, D-69117 Heidelberg, Germany; meiert.grootes@mpi-hd.mpg.de \\ 2 Jeremiah Horrocks Institute, University of Central Lancashire, Preston PR1 2HE, UK \\ ${ }^{3}$ Sydney Institute for Astronomy, School of Physics, University of Sydney, NSW 206, Australia \\ ${ }^{4}$ Scottish Universities' Physics Alliance (SUPA), School of Physics and Astronomy, University of St. Andrews, North Haugh, St. Andrews KY16 9SS, UK \\ ${ }^{5}$ International Centre for Radio Astronomy Research (ICRAR), University of Western Australia, Stirling Highway Crawley, WA 6009, Australia \\ ${ }^{6}$ European Southern Observatory, Karl-Schwarzschild Str. 2, D-85748 Garching, Germany \\ ${ }^{7}$ Observatories of the Carnegie Institution for Science, 813 Santa Barbara Street, Pasadena, CA 91101, USA \\ ${ }^{8}$ Centre for Astrophysics and Supercomputing, Swinburne University of Technology, Hawthorn, Victoria 3122, Australia \\ ${ }^{9}$ Sterrenkundig Observatorium, Universiteit Gent, Krijgslaan 281 S9, B-9000 Gent, Belgium \\ ${ }^{10}$ Astrophysics Research Institute, Liverpool John Moores University, Twelve Quays House, Egerton Wharf, Birkenhead CH41 1LD, UK \\ ${ }^{11}$ Centre for Astronomy and Particle Theory, The School of Physics \& Astronomy, Nottingham University, University Park Campus, Nottingham NG7 2RD, UK \\ ${ }^{12}$ Australian Astronomical Observatory, P.O. Box 296, Epping, NSW 1710, Australia \\ ${ }^{13}$ Department of Physics and Astronomy, University of California, Irvine, CA 92697, USA \\ ${ }^{14}$ Physics Department, Imperial College, Prince Consort Road, London SW7 2AZ, UK \\ ${ }^{15}$ INAF-Osservatorio Astronomico di Padova, Vicolo Osservatorio 5, I-35122 Padova, Italy \\ ${ }^{16}$ SISSA, Via Bonomea 265, I-34136 Trieste, Italy \\ ${ }^{17}$ Department of Physics and Astronomy, University of Canterbury, Private Bag 4800, Christchurch 8140, New Zealand \\ ${ }^{18}$ School of Physics \& Astronomy, Cardiff University, Queen Buildings, The Parade, Cardiff CF24 3AA, UK \\ ${ }^{19}$ Department of Physical Sciences, The Open University, Milton Keynes MK7 6AA, UK \\ ${ }^{20}$ Centre for Astrophysics Research, Science \& Technology Research Institute, University of Hertfordshire, Hatfield, Herts AL10 9AB, UK \\ ${ }^{21}$ Physics Department University of the Western Cape, Cape Town 7535, South Africa \\ ${ }_{22}$ Astronomy Centre, University of Sussex, Falmer, Brighton BN1 9QH, UK \\ ${ }^{23}$ Institute for Astronomy, University of Edinburgh, Royal Observatory, Blackford Hill, Edinburgh EH9 3HJ, UK \\ ${ }^{24}$ Institute for Computational Cosmology, Department of Physics, Durham University, Durham DH1 3LE, UK \\ ${ }^{25}$ Institute of Cosmology and Gravitation, Portsmouth University, Dennis Sciama Building, Portsmouth PO1 3FX, UK \\ Received 2012 February 24; accepted 2013 January 30; published 2013 March 7
}

\begin{abstract}
We report the discovery of a well-defined correlation between $B$-band face-on central optical depth due to dust, $\tau_{B}^{f}$, and the stellar mass surface density, $\mu_{*}$, of nearby $(z \leqslant 0.13)$ spiral galaxies: $\log \left(\tau_{B}^{f}\right)=1.12( \pm 0.11)$. $\log \left(\mu_{*} / M_{\odot} \mathrm{kpc}^{-2}\right)-8.6( \pm 0.8)$. This relation was derived from a sample of spiral galaxies taken from the Galaxy and Mass Assembly (GAMA) survey, which were detected in the FIR/submillimeter (submm) in the HerschelATLAS science demonstration phase field. Using a quantitative analysis of the NUV attenuation-inclination relation for complete samples of GAMA spirals categorized according to stellar mass surface density, we demonstrate that this correlation can be used to statistically correct for dust attenuation purely on the basis of optical photometry and Sérsic-profile morphological fits. Considered together with previously established empirical relationships of stellar mass to metallicity and gas mass, the near linearity and high constant of proportionality of the $\tau_{B}^{f}-\mu_{*}$ relation disfavors a stellar origin for the bulk of refractory grains in spiral galaxies, instead being consistent with the existence of a ubiquitous and very rapid mechanism for the growth of dust in the interstellar medium. We use the $\tau_{B}^{f}-\mu_{*}$ relation in conjunction with the radiation transfer model for spiral galaxies of Popescu \& Tuffs to derive intrinsic scaling relations between specific star formation rate (SFR), stellar mass, and stellar surface density, in which attenuation of the UV light used for the measurement of SFR is corrected on an object-to-object basis. A marked reduction in scatter in these relations is achieved which we demonstrate is due to correction of both the inclination-dependent and face-on components of attenuation. Our results are consistent with a general picture of spiral galaxies in which most of the submm emission originates from grains residing in translucent structures, exposed to UV in the diffuse interstellar radiation field.
\end{abstract}

Key words: dust, extinction - galaxies: fundamental parameters - galaxies: ISM - galaxies: spiral

\section{INTRODUCTION}

Broadband photometric imaging surveys in the UV/optical (e.g., SDSS (Abazajian et al. 2009), KiDS (de Jong et al. 2012), EUCLID (Laureijs et al. 2011), GALEX MIS) are, and will continue to be, a main source of information from which the physical properties of galaxies must be deduced. It is, however, a well-known issue that the UV/optical emission of galaxies is strongly attenuated by dust and that this attenuation should be taken into account (e.g., Driver et al. 2007; Masters et al. 2010). This is particularly the case for late-type galaxies, which are usually much more gas- and dust-rich than early-type galaxies (as recently re-confirmed using Herschel data by, e.g., Rowlands et al. 2011; Dariush et al. 2011; Bourne et al. 2012). 
Furthermore, detailed imaging studies of dust emission in the Milky Way and nearby spiral galaxies (e.g., Molinari et al. 2010; Bendo et al. 2011; Braine et al. 2010; Fritz et al. 2012) show that most of the dust is associated with large-scale structures in the neutral and molecular gas layers, which in turn causes the attenuation to depend heavily on disk inclination (Tuffs et al. 2004; Pierini et al. 2004; Driver et al. 2007).

While it is generally agreed that the UV/optical emission of late-type galaxies must be corrected for dust attenuation, it has proven to be a challenge to measure the opacities of the disks, and various approaches exist. The most powerful method is to utilize infrared measurements of dust emission in combination with UV/optical data, since the attenuating dust is heated by the $\mathrm{UV} /$ optical emission it absorbs, and the bulk of this energy is re-radiated longward of $60 \mu \mathrm{m}$ in the far-infrared (FIR) and submillimeter (submm) spectral range. Approaches utilizing this UV/optical-FIR/submm information range from semiempirical ones, such as the infrared excess absorption estimator (Meurer et al. 1999; Seibert et al. 2005; Johnson et al. 2007), via spectral energy distribution (SED) fitting using energy balance considerations (da Cunha et al. 2008; Noll et al. 2009; Serra et al. 2011), to radiation transfer modeling approaches, which explicitly calculate the UV/optical illumination of dust and the resulting FIR/submm emission (Silva et al. 1998; Popescu et al. 2000, 2011; Bianchi et al. 2000; Gordon et al. 2001; Misselt et al. 2001; Bianchi 2008; Baes et al. 2010, 2011; MacLachlan et al. 2011, hereafter PT11). Unfortunately, such a coverage of the full UV/optical-FIR/submm SED is seldom or only incompletely available for the population of spiral galaxies (despite their significant dust opacities) due to the scarcity of wide and sufficiently deep FIR surveys. Consequently, these methods can often only be applied to more massive spiral galaxies and starbursts.

In the absence of FIR data, semi-empirical models, based solely on UV/optical data, such as the UV spectral slope $\beta$ or the Balmer decrements, are often applied (Cardelli et al. 1989; Meurer et al. 1999; Calzetti 2001; Kong et al. 2004; Seibert et al. 2005; Wijesinghe et al. 2011). These, however, often depend on either multiple UV bands or spectroscopy, information which is also often unavailable.

In this paper, we present a correlation between the $B$-band face-on central dust-opacity $\tau_{B}^{f}$ and the stellar mass surface density $\mu_{*}$ of late-type galaxies without evidence of the presence of an active galactic nucleus (AGN), and demonstrate that this can be used in combination with the radiation transfer model of PT11 to statistically correct samples of late-type galaxies for the attenuation due to dust using only broadband optical photometry. The basis for this analysis is the overlap between the UV-optical-NIR/spectroscopic Galaxy and Mass Assembly (GAMA) survey (GAMA; Driver et al. 2011), and the FIR/submm Herschel-ATLAS (H-ATLAS; Eales et al. 2010) survey. After briefly recapitulating some of the key concepts of the PT11 model (Section 2) and describing the data and samples employed (Section 3), we extract direct values of $\tau_{B}^{f}$ from the UV/optical-FIR data for a subsample of local universe late-type galaxies jointly measured by GAMA and H-ATLAS in the H-ATLAS science demonstration phase (SDP) field and use these to derive and calibrate the correlation between $\tau_{B}^{f}$ and $\mu_{*}$, as described in Section 4. In Section 5, we show that the correlation correctly predicts the NUV attenuation-inclination relations for complete subsamples of GAMA late-type galaxies characterized in terms of $\mu_{*}$, thus demonstrating its predictive power to correct samples of late-type galaxies without available
FIR data for dust attenuation using only broadband optical photometry. We then discuss our findings in the context of the production/injection/survival of dust in spiral galaxies and the scaling relations between specific star formation rate (SFR), stellar mass, and stellar mass surface density in Section 6, and summarize our results in Section 7. We use AB magnitudes throughout this analysis, and adopt an $H_{0}=70 \mathrm{~km} \mathrm{~s}^{-1} \mathrm{Mpc}^{-1}$, $\Omega_{M}=0.3, \Omega_{\Lambda}=0.7$ cosmology (Spergel et al. 2003).

\section{THE RADIATION TRANSFER MODEL}

In this paper, we quantitatively link the characteristics of the attenuation of starlight in spiral galaxies to the mass of dust in the galaxies as directly determined from the FIR/submm integrated photometry. This approach mandates assumptions about the spatial distribution of dust in the galaxies. Here, we utilize the radiation transfer model of Popescu et al. (2011, hereafter PT11), which is applicable to a wide range of nonstarburst, late-type galaxies. We refer the reader to PT11 as well as Popescu et al. (2000) and Tuffs et al. (2004) for a detailed description of the model, its parameters, and the work done to test its performance. Here, we only supply a brief summary and detail its application to our data. The model consists of a bulge with an old stellar population and two exponential disks describing the distribution of old and young stellar populations as well as of diffuse dust located in these disks. This diffuse dust component can be seen as representing dusty structures (such as Cirrus) with a substantial projected surface filling factor. Emission from a dustless bulge is parameterized through the inclusion of a bulge-to-disk ratio (B/D) to accommodate a range of geometries along the Hubble sequence. In addition, the model includes a clumpy dust component with an embedded young stellar population representing star-forming regions. The fraction of UV emission escaping from the regions into the diffuse interstellar medium (ISM) is given by a factor $1-F$ (fixed to $F=0.41$ for this analysis, following PT11). This model, specifically the relative scale lengths and scale heights of the stars and diffuse dust in the exponential disks, has been calibrated on and fixed to the reproducible trends found in the local edge-on spiral galaxies analyzed in the radiation transfer analysis of Xilouris et al. (1999). As such, the wavelength dependence of the scale lengths is also fixed.

In the PT11 model, the opacity of the disk at a given frequency and position can be expressed in terms of the central face-on optical depth of the combination of the two dust disks at a reference wavelength (PT11 use the $B$ band at $4430 \AA$ ),$\tau_{B}^{f}$. The value of $\tau_{B}^{f}$ can be expressed as

$$
\tau_{B}^{f}=K \frac{M_{\mathrm{dust}}}{r_{s, d, B}^{2}},
$$

where $M_{\text {dust }}$ is the total mass of dust in the galaxy, $r_{s, d, B}$ is the scale length of the exponential disk in the $B$ band, and $K$ is a constant combining the fixed large-scale geometry and the spectral emissivity of the Weingartner \& Draine (2001) model. For the purposes of the work presented here the value of $\tau_{B}^{f}$ must be derived from observable properties, hence Equation (1) must be re-expressed in terms of observational quantities.

With the geometry of the model fixed, we express the physical scale length of the disk at the reference wavelength, $r_{s, d, B}$, using the corresponding angular size at the redshift at which the source is observed. We have chosen to determine this angular size in the $r$ band, which is less affected by the effects of dust attenuation 
than shorter wavelengths, while being less affected by noise than longer passbands, in particular the NIR, which may also suffer from systematic uncertainties (cf., e.g., Taylor et al. 2011).

The mass of dust $M_{\text {dust }}$ is determined from the FIR/submm observations available from H-ATLAS. These data extend longward of $100 \mu \mathrm{m}$, thus predominantly sampling the emission by cold dust in the galaxy and warranting the assumption that this range of the SED can be reasonably approximated by a modified Planckian $S_{v}(v) \sim v^{\beta} B(v, T)$ with $\beta=2$ (i.e., the dust emission is not heavily affected by a warm dust component and/or stochastic heating processes broadening the peak of the SED). This allows Equation (1) to be re-expressed as

$$
\tau_{B}^{f}=A \frac{(1+z)^{3-\beta}}{B\left((1+z) v_{250}, T_{0}\right)} \frac{S_{\nu}\left(v_{250}\right)}{\theta_{s, d, r}^{2}},
$$

with $A=6.939 \times 10^{-13} \operatorname{arcsec}^{2} \mathrm{~J} \mathrm{Jy}^{-1} \mathrm{~s}^{-1} \mathrm{~Hz}^{-1} \mathrm{~m}^{-2} \mathrm{sr}^{-1}, \theta_{s, d}, r$ representing the $r$-band angular size corresponding to the disk scale length in arcseconds, $S_{v}\left(\nu_{250}\right)$ representing the observed monochromatic flux density at $250 \mu \mathrm{m}$ in $\mathrm{Jy}$, and $B(\nu, T)$ representing a Planckian with units of $\mathrm{W} \mathrm{Hz}^{-1} \mathrm{~m}^{-2} \mathrm{sr}^{-1}$, with a rest-frame temperature $T_{0} . T_{0}, S_{v}\left(v_{250}\right)$, and $\theta_{s, d, r}$ will be derived from measurements of spatially integrated FIR/submm SEDs and optical morphologies in Section 4. The numerical value of $A$ has been calibrated using the detailed radiation transfer analysis results of the Xilouris et al. (1999) galaxy sample. A detailed derivation of Equations (1) and (2) and their link to the PT11 model together with a detailed description of the numerical calibration of $A$ is provided in Appendix A.

The choice of using $250 \mu \mathrm{m}$ is motivated by the tradeoff between using a measurement as far in the FIR/submm as possible, thus dominated by thermal emission of cold dust, and the sensitivity of the available data as discussed in Section 3. The values of $\tau_{B}^{f}$ depend somewhat on the fitted rest-frame temperatures of the modified Planckian fits via the temperature dependence of the derived dust masses as shown in Section 4.1; the typical uncertainty in the temperature of $\sim 1 \mathrm{~K}$ corresponds to an uncertainty of the dust mass of $\sim 15 \%$.

\section{DATA AND SAMPLES}

The GAMA survey, currently comprising 11 band UV-NIR photometry (FUV, NUV, $u, g, r, i, z, Y, J, H, K$ ), and $300-800 \mathrm{~nm}$ optical spectroscopy covering $\approx 144 \mathrm{deg}^{2}$ to $r_{\text {petro }}<19.4$ mag with a spectroscopic completeness of $>96 \%$, provides a large, statistically complete, sample of local universe galaxies with homogeneous photometry, spectroscopy, and ancillary data. This paper makes particular use of the stellar masses provided by Taylor et al. (2011) and single Sérsic-profile fits performed by Kelvin et al. (2012), providing morphological information. For a full description of the GAMA survey see Driver et al. (2011) as well as Baldry et al. (2010) for details of the input catalog, Robotham et al. (2010) for details of the high-completeness tiling scheme, and Hill et al. (2011) together with E. Andrae et al. (in preparation) for details of the photometry. By design, GAMA's coverage in the UV-optical-NIR is complemented in the FIR by the H-ATLAS survey (Eales et al. 2010) using the PACS (Poglitsch et al. 2010) and SPIRE (Griffin et al. 2010) instruments on board the Herschel Space Observatory (Pilbratt et al. 2010). H-ATLAS achieves $5 \sigma$ point source sensitivities of $132,126,32,26$, and $45 \mathrm{mJy}$ in the $100 \mu \mathrm{m}$, $160 \mu \mathrm{m}, 250 \mu \mathrm{m}, 350 \mu \mathrm{m}$, and $500 \mu \mathrm{m}$ channels, respectively. The details of the SPIRE and PACS map-making process are described in Pascale et al. (2011) and Ibar et al. (2010), while the

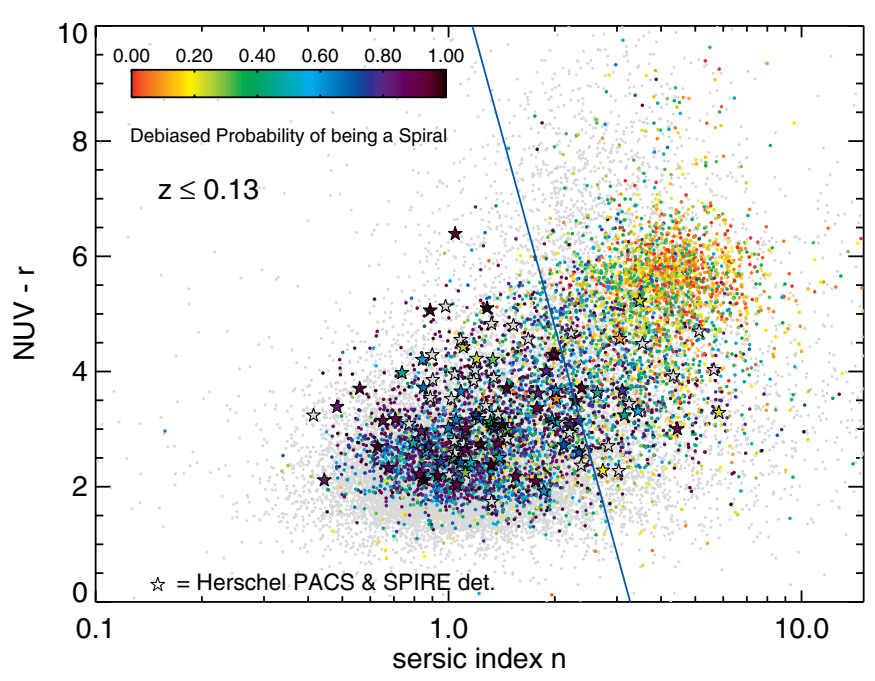

Figure 1. NUV $-r$ vs. $n_{r}$ for non-AGN GAMA galaxies $(z \leqslant 0.13)$ with NUV and $r$-band detections. The color-coded subsample represents the overlap with GALAXY ZOO DR1 sources with debiased morphological classifications (Lintott et al. 2008; Bamford et al. 2009) with the color representing the probability that the source is a spiral. The blue line represents an automated morphological selection designed to reliably select late-type galaxies with minimal contamination, calibrated on the GALAXY ZOO data (see GR12 for details). Overplotted as stars are the sources detected in the H-ATLAS SDP field with SPIRE and PACS, with those in OPTICAL+FIR located left of the divide.

catalogs are described in Rigby et al. (2010). The FIR data used in this analysis are taken from the catalog of H-ATLAS sources matched to optical SDSS/GAMA sources using a likelihoodratio method (Smith et al. 2011), with a required reliability of $>80 \%$. This catalog is defined by the requirement of $5 \sigma$ detections at $250 \mu \mathrm{m}$.

In the context of this work, it is critical to select normal, i.e., non-starburst, non-AGN, late-type galaxies as reliably as possible, as the radiation transfer model employed is designed to treat, and is calibrated on, such sources, and is therefore only accurate for these. We first select those GAMA sources which have NUV and $r$-band detections, reliable spectroscopic redshifts, and which have not been identified as AGNs following the prescription of Kewley et al. (2006), ${ }^{26}$ limiting ourselves to a local universe sample with $z \leqslant 0.13$ to avoid evolutionary effects. $^{27}$ From these 14998 galaxies, we select a population of probable spiral galaxies in the NUV $-r$ versus $r$-band Sérsic index $n_{r}$ plane (Driver et al. 2012; Kelvin et al. 2012) as those with

$$
(\mathrm{NUV}-r) \leqslant-22.35 \log \left(n_{r}\right)+11.5
$$

resulting in a subset of 11,236 galaxies which we will refer to as the OPTICAL sample. The separator defined by Equation (3) corresponds to the blue line in Figure 1. It was determined from GAMA sources with debiased visual morphological classifications from GALAXY ZOO DR1 (Lintott et al. 2008; Bamford et al. 2009), and is designed to maximize the number of GALAXY ZOO spirals correctly classified while simultaneously minimizing the contamination of the spiral sample due to GALAXY ZOO ellipticals based on a figure of merit maximization approach. As such, it is a conservative classification and will exclude some real spirals. We find a contamination

\footnotetext{
26 Almost all these sources have available Sérsic parameters and stellar mass estimates. Those that do not are excluded from the sample.

27 This limitation in redshift also is conducive to our confidence in the derived values of the Sérsic parameter $n$, as meaningful morphological fits become more difficult at larger redshifts.
} 
of the resulting spiral sample by reliably classified ellipticals of $5 \%-10 \%$. From OPTICAL, we select the subset of sources with $>3 \sigma$ detections in the $100 \mu \mathrm{m}$ and/or $160 \mu \mathrm{m}$ channel in addition to at least a $5 \sigma$ detection in the $250 \mu \mathrm{m}$ channel (i.e., from the H-ATLAS SDP field where both SPIRE and PACS catalogs are currently available). We then calculate values of $\tau_{B}^{f}$ for these 79 sources as described in Section 4 excluding two with $\tau_{B}^{f}>30$ (which we take to be indicative of starburst systems). Finally, we exclude three sources which have been visually classified as early-type galaxies by Rowlands et al. (2011), resulting in a sample of 74 late-type galaxies with detections in at least two FIR bands, referred to as the OPTICAL+FIR sample.

\section{THE OPACITY-SURFACE DENSITY CORRELATION}

\subsection{Deriving Opacities}

For each of the galaxies in the OPTICAL+FIR sample, the disk opacity was calculated from Equation (2) using knowledge of $T_{0}$ and $S_{250}$ (derived from Herschel data), and the $r$-band angular exponential disk scale $\theta_{s, d, r}$ (derived from $\theta_{e, \mathrm{ss}, r}$ the $r$-band single Sérsic effective size in arcsec (i.e., the half-light radius) cataloged by Kelvin et al. 2012).

To derive $T_{0}$ from the Herschel data, we fit isothermal modified Planckians $(\beta=2)$ to all available data points. The requirement of a detection at $160 \mu \mathrm{m}$ or shortward allows the spectral peak of the dust emission to be well constrained. We find a median value of $23.2 \mathrm{~K}$ for $T_{0}$. The value of $T_{0}$ is almost independent of the wavelengths at which the Herschel data are obtained, as the median temperature of sources with only a $160 \mu \mathrm{m}$ PACS detection is $22.58 \mathrm{~K}$, while that of sources with a PACS detection only at $100 \mu \mathrm{m}$ is $23.58 \mathrm{~K}$. The median temperature of sources with PACS detections at both $100 \mu \mathrm{m}$ and $160 \mu \mathrm{m}$ is $23.35 \mathrm{~K}$. The difference in median dust temperature of galaxies between the $100 \mu \mathrm{m}$ only and the $160 \mu \mathrm{m}$ only sample corresponds to an uncertainty in the dust mass of $\approx 15 \%$. The requirement of a data point at $160 \mu \mathrm{m}$ or shortward does not appear to induce a strong bias toward warmer sources, since the median temperature of our sample is consistent with the mean value of $22.7 \pm 2.9 \mathrm{~K}$ for blue galaxies with $3 \times 10^{9}<M_{*}<3 \times 10^{11} M_{\odot}$ (roughly comparable to our sample) found by Bourne et al. (2012) using a stacking analysis of H-ATLAS data on optically selected galaxies.

Overall, we believe that the isothermal model constrained by SPIRE data at $\lambda \geqslant 250 \mu \mathrm{m}$ and a PACS data point at 100 and/or $160 \mu \mathrm{m}$ represents a robust method of determining dust masses using minimal assumptions, due to the decrease in the uncertainty of both temperature and amplitude arising from a data point constraining the peak of the dust emission, and because the wavelength coverage $(\lambda>100 \mu \mathrm{m})$ misses any significant emission arising from warm dust in SF regions or from stochastically heated small grains in the diffuse ISM (e.g., Popescu et al. 2000; Gordon et al. 2001; Misselt et al. 2001; Galliano et al. 2003, 2005).

Using Equation (2) to determine $\tau_{B}^{f}$ requires knowledge of the angular size corresponding to the disk scale length in the $r$ band. The relation between the observable single Sérsic effective size and the disk scale-length of a spiral galaxy, however, is nontrivially influenced by the relative fraction of emission from the bulge and the disk as well as by dust present in the galaxy, with the former causing the ratio between $\theta_{e, \mathrm{ss}, r}$ and $\theta_{s, d, r}$ to decline, while the latter tends to cause sizes to be overestimated, increasing the ratio. B. Pastrav et al. (in preparation) have investigated the combined dependencies of the ratio between $\theta_{e, \mathrm{ss}, r}$ and $\theta_{s, d, r}$ on bulge-to-disk ratio, dust opacity, inclination, and wavelength, and provide their results in tabulated form. In this work, we have self-consistently determined $\tau_{B}^{f}$ for the $O P T I C A L+F I R$ sample using Equation (2) and the results of Pastrav et al. as detailed in Appendix B. In doing so, we have used a bulge-to-disk ratio of $B / D=0.33$ found to be representative of the generally earlier type, more massive spirals (Graham \& Worley 2008), such as those in the OPTICAL+FIR sample. We caution that this use of an average value of $B / D$ will introduce uncertainty into the derived values of $\tau_{B}^{f}$ as shown in Appendix B, and will revisit our results when reliable bulge + disk decompositions based on higher resolution imaging of these objects become available.

\subsection{Deriving Stellar Mass Surface Densities}

We compute the stellar mass surface density $\mu_{*}$ using the physical radius $r_{e, \mathrm{ss}, r}$ corresponding to the single Sérsic effective radius in arcsec provided by Kelvin et al. (2012) and the GAMA stellar masses $M_{*}$ from Taylor et al. (2011) as

$$
\mu_{*}=\frac{M_{*}}{2 \pi r_{e, \mathrm{ss}, r}^{2}}=\frac{M_{*}}{2 \pi D_{A}^{2}(z) \theta_{e, \mathrm{ss}, r}^{2}},
$$

where $D_{A}^{2}(z)$ is the angular diameter distance corresponding to the redshift $z$. We note that the stellar masses predicted by Taylor et al. incorporate a single fixed prediction of the reddening and attenuation due to dust derived from Calzetti et al. (2000). Thus, expected systematic variations in reddening and attenuation with inclination, disk opacity, and bulge-to-disk ratio are not taken into account. However, as discussed by Taylor et al. (see also Figure 12 of Driver et al. 2007) the resulting shifts in estimated stellar mass are much smaller than the individual effects on color and luminosity, and should not significantly affect the relation between disk opacity and stellar mass surface density derived in this paper. Taking this, and other effects into account, the typical uncertainty in the stellar mass estimated by Taylor et al. is $\sim 0.1$ dex.

We also note that Taylor et al. (2011) make use of a Chabrier (2003) initial mass function (IMF) and the Bruzual \& Charlot (2003) stellar population library, and that hence, any systematic variations due to the choice of IMF or the stellar population library are not taken into account.

\subsection{The Relation between Opacity and Stellar Mass Surface Density}

$\tau_{B}^{f}$ is plotted against $\mu_{*}$ for the $O P T I C A L+F I R$ sample in Figure 2. The data points are shown as symbols according to their Sérsic index, with the color corresponding to the NUV $-r$ color. Using a linear regression analysis taking the uncertainties in both $\mu_{*}$ and $\tau_{B}^{f}$ into account, we find a power-law correlation between the two with $\chi^{2} / N_{\mathrm{DOF}}=0.97\left(N_{\mathrm{DOF}}=72\right)$ as

$$
\log \left(\tau_{B}^{f}\right)=1.12( \pm 0.11) \cdot \log \left(\frac{\mu_{*}}{M_{\odot} \mathrm{kpc}^{-2}}\right)-8.6( \pm 0.8)
$$

depicted by the dash-dotted line in Figure 2. The errors represent the $1 \sigma$ uncertainties in the regression analysis. The correlation is tightest for sources with NUV $-r$ colors of $\sim 3$ with a slight increase in scatter for bluer and redder colors. This increase is likely due in part to the assumed $\mathrm{B} / \mathrm{D}$ ratio in the determination of $\tau_{B}^{f}$ as detailed in Appendix B, but may also represent 


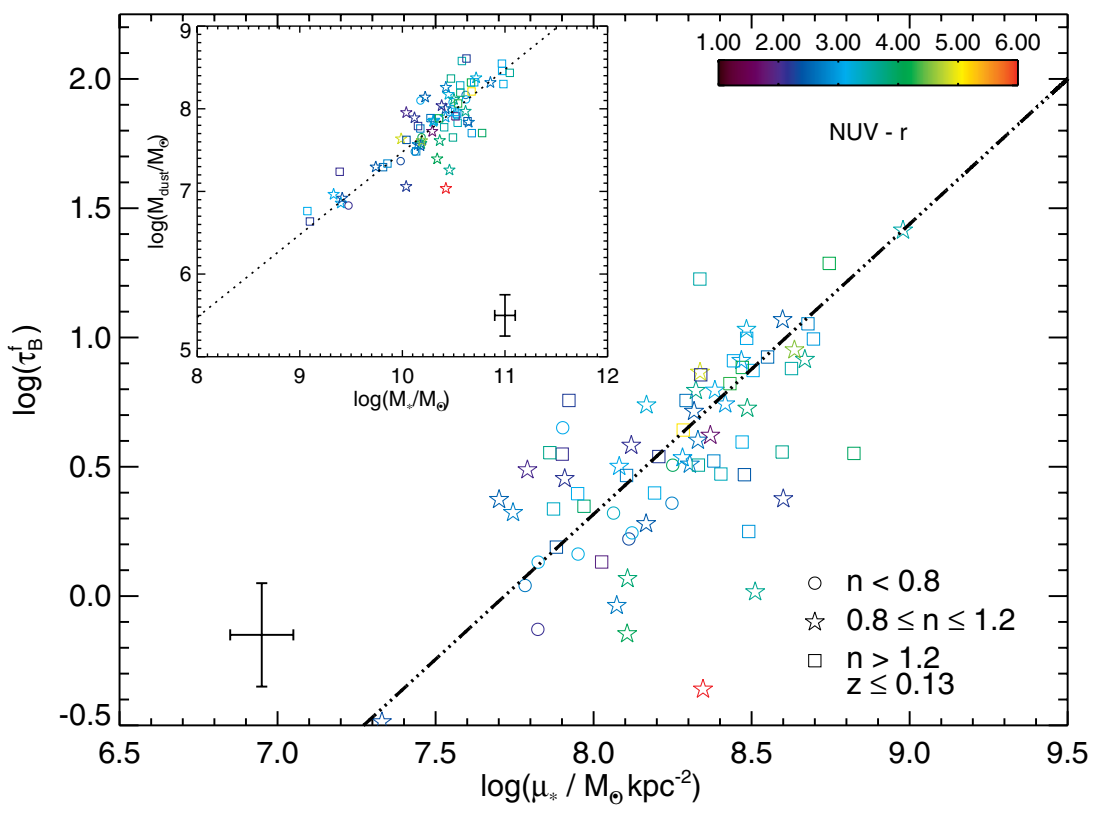

Figure 2. $B$-band face-on central optical depth $\tau_{B}^{f}$ vs. stellar mass surface density $\mu_{*}$ for $O P T I C A L+F I R$ galaxies. Symbols are coded according to $n$ and NUV-r color (see figure). The dash-dotted line represents the best fit (Equation (5)). The median uncertainties in $\tau_{B}^{f}$ and $\mu_{*}$ are depicted at bottom left. The inset depicts the dust mass (derived from $\tau_{b}^{f}$ using Equations (1) and (2)) as a function of stellar mass. The dotted line represents a reference value with a slope of unity and an offset corresponding to $M_{\text {dust }} / M_{*}=0.003$. Median errors are depicted bottom right.

a larger range in opacities for bluer possibly more irregular system and redder systems which may appear red either due to dust, or because they are more passive systems. There is also evidence for such a population of passive spirals, i.e., spirals with low $\tau_{B}^{f}$ high $\mu_{*}$, as presented by Rowlands et al. (2011). We find that $50 \%$ of the sample lie within 0.14 dex of the correlation $\left(\Delta_{s, 0.5} \log \left(\tau_{B}^{f}\right)=0.14\right)$, comparable to the median measurement error for $\log \left(\tau_{B}^{f}\right)$. Thus, it is possible that a large fraction of the visible scatter is due to measurement uncertainties.

\subsubsection{Range of Applicability and Limitations of the Relation}

In order to understand the range of applicability of the correlation shown in Figure 2 and given by Equation (5), as well as to identify possible biases caused by the use of an FIR-selected sample in deriving this result, we have overplotted the distribution of the OPTICAL+FIR sample in the $\mu_{*}$ versus $M_{*}$ plane on that of the OPTICAL sample in Figure 3 . The $O P T I C A L+F I R$ sample covers a range of $7.6<\log \left(\mu_{*}\right)<9.0$ in $\mu_{*}$ more or less uniformly and can be deemed applicable in this range. Figure 3, however, also clearly shows that the $O P T I C A L+F I R$ sample is strongly biased toward more massive sources, as shown by the positions of the purple stars. This bias arises from the fact that the OPTICAL+FIR is defined by the sensitivity of the Herschel instruments. In spite of this clear bias in stellar mass, however, the sample does contain sources which provide a tentative coverage of the entire stellar mass range corresponding to the range in $\mu_{*}$ as seen in the OPTICAL sample. As discussed in Section 5, this bias in stellar mass does not affect the applicability of Equation (5) to large samples of galaxies as constituted by the OPTICAL sample.

The range of $7.6<\log \left(\mu_{*}\right)<9.0$ for which Equation (5) may be deemed applicable only provides a complete sample of galaxy stellar mass above $10^{9.5} M_{\odot}$. Thus, if the correlation is to be applied to samples of galaxies which need to be complete in stellar mass, these should be accordingly limited until the correlation can be calibrated for lower values of $\mu_{*}$ (corresponding also to lower values of $M_{*}$ ).

Figure 3 also shows that $\mu_{*}$ appears to be loosely correlated with $M_{*}$, with higher mass galaxies having larger values of $\mu_{*}$. This raises the question of whether the $\tau_{B}^{f}-\mu_{*}$ correlation shown in Figure 2 is actually a relation between $\tau_{B}^{f}$ and $M_{*}$. Using a linear partial correlation analysis of $X=\log \left(M_{*}\right), Y=\log \left(\mu_{*}\right)$, and $Z=\log \left(\tau_{B}^{f}\right)$ on the $O P T I C A L+F I R$ sample, we obtain the partial correlation coefficients $r_{X Y, Z}=0.271, r_{X Z, Y}=-0.047$, and $r_{Y Z, X}=0.611$, however, implying that $\mu_{*}$ is indeed the dominant factor in determining $\tau_{B}^{f}$.

Furthermore, as Equation (5) presents a relation between two properties which are both inversely proportional to an area, we must ask ourselves whether the result is actually a spurious correlation due to noise in the size measurements. Due to the moderate redshift limit of $z \leqslant 0.13$, however, the uncertainties on the size determination are much smaller than the range in sizes found for a given value of $M_{*}$, showing that the spread in values of $\mu_{*}$ is mainly intrinsic.

Finally, we emphasize that the quantitative accuracy of the relation given by Equation (5) depends on the applicability of the large-scale geometry of the exponential dust disks as calibrated in PT11 to the range of late-type galaxies with $7.6<\log \left(\mu_{*}\right)<9.0$.

\subsection{The Dust-Mass-to-Stellar-Mass Relation}

It is clear that $\tau_{B}^{f}$ is akin to a surface density and requires measurements of both a galaxy's stellar mass and size to facilitate it's estimation. Nevertheless, a major underlying physical driver for the result presented by Equation (5) is a roughly linear correlation between the mass of dust and stars in late-type galaxies of the OPTICAL+FIR sample. This is shown in the inset of Figure 2, where dust masses (derived from the values of $\tau_{B}^{f}$ using Equation (1)) are plotted against stellar masses from 


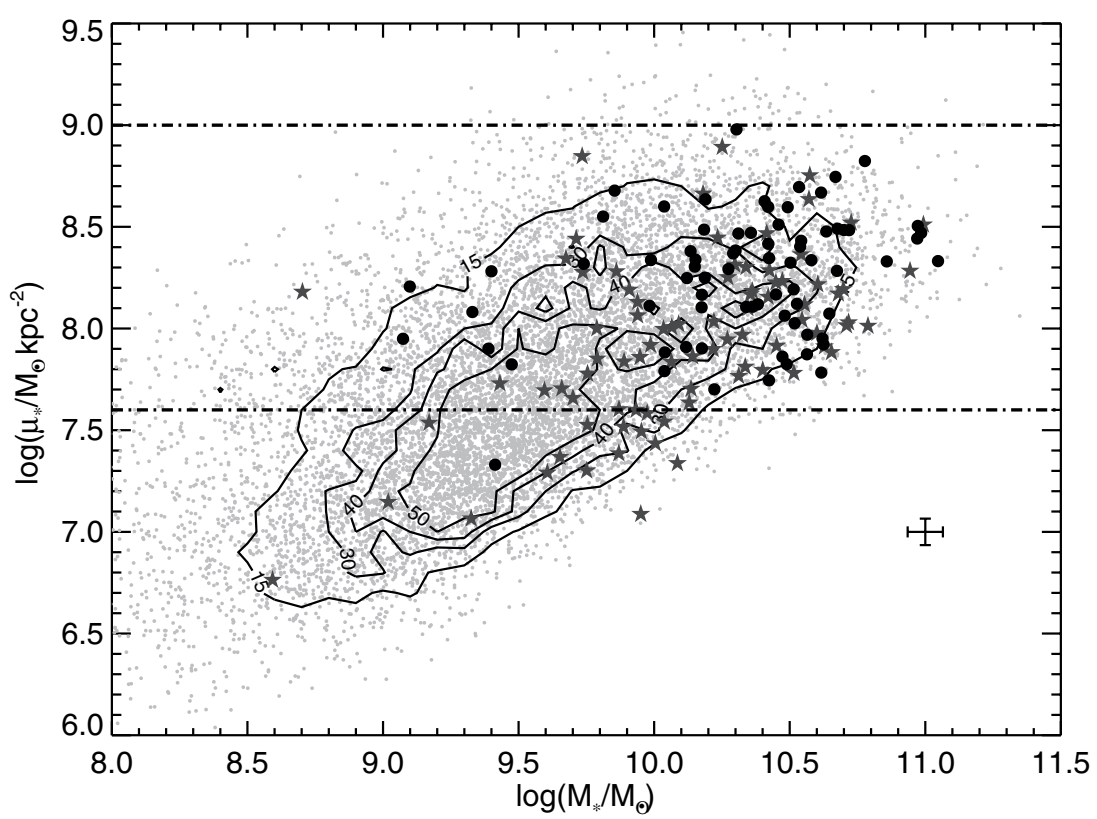

Figure 3. $\mu_{*}$ as a function of $M_{*}$ for $O P T I C A L$ (gray) galaxies with isodensity contours. Overplotted are the $O P T I C A L+F I R$ sources (circles) and those sources which fulfill the morphological selection but only have SPIRE detections (stars). Dash-dotted lines indicate the range in $\mu_{*}$ for which the correlation has been calibrated. The median errors on both properties are shown at bottom right.

Taylor et al. (2011). The dotted line depicts a slope of unity with a dust-to-stellar mass fraction of $3 \times 10^{-3}$ as a reference value.

\subsubsection{Comparison with Other Herschel Results}

Several previous works have provided data on the dust-tostellar mass ratio for different samples of galaxies, allowing quantitative comparisons with our results. Skibba et al. (2011) present stellar and dust masses for the galaxies in the Herschel KINGFISH survey (Kennicutt et al. 2011). For a sample of spiral galaxies of type Sa and later with $M_{*}>10^{9} M_{\odot}$, comparable to our sample, one finds an average dust-to-stellar mass ratio of $-3.02 \pm 0.5$ (derived from Table 1 of Skibba et al. 2011), comparable within errors to our result. Furthermore the dustto-stellar mass ratio inferred by our data is comparable within errors to that found for spiral galaxies in the Herschel Reference Survey (HRS; Boselli et al. 2010) by Cortese et al. (2012), as shown in Figure 5 of Cortese et al. (2012) for individual morphological types and in Figure 9 of Smith et al. (2012) for all spiral galaxies in the HRS. The agreement is particularly good for the dust-to-stellar mass ratios derived for earlier-type spirals which, on average, are more massive and are likely to be more directly comparable to our sample (Cortese et al. 2012, their Figure 5). In addition Figure 5 of Cortese et al. (2012) also shows that the dust-to-stellar mass ratio is nearly constant for galaxies with morphological type Sa and later, especially for galaxies with $\mathrm{H}$ I deficiencies generally indicative of residing in environments comparable to our sample (i.e., not being members of massive clusters).

Finally, we find that the dust-to-stellar mass ratio of $\sim 3 \times$ $10^{-3}$, is also in general agreement with that derived by Dunne et al. (2011; $2 \times 10^{-3}$ ) for low redshift galaxies using all H-ATLAS SDP field sources, although we note that these authors have employed a different calibration of FIR/submm dust emissivity.

The roughly linear slope of the relation between dust and stellar mass in the OPTICAL+FIR sample also generally agrees well with the data for late-type HRS galaxies plotted in Figure 8 of Smith et al. (2012). The data show a slope which is slightly sublinear over a large range in stellar mass extending down to below $10^{9} M_{\odot}$. At galaxy stellar masses above $10^{9.5} M_{\odot}$ (more similar to our OPTICAL+FIR sample), however, the data exhibit a slope which is considerably closer to unity. The results of Bourne et al. (2012), who find a correlation between dust and stellar mass based on a stacking analysis of optically selected sources, display similar properties, with the relation between dust and stellar mass steepening with increasing stellar mass (their Figure 16). We note that Bourne et al. (2012) do not apply a morphological classification, but rather categorize their sample into blue, green, and red bins according $g-r$ color which is likely to place some of the dusty edge-on spirals included in our sample in the green or even red bin in which their data display a steeper, more linear slope.

\section{TESTING THE PREDICTIVE POWER OF THE STELLAR MASS SURFACE DENSITY-OPACITY RELATION}

The $\tau_{B}^{f}-\mu_{*}$ relation as formulated in Equation (5) is fundamentally an empirical result, linking the total dust mass per directly measured area to a direct measure of the stellar mass per unit area. Thus, the relation is largely independent of detailed assumptions about the distribution of dust within the galaxy disks. Nevertheless, since we have formulated the previous analysis in the terms of the $\tau_{B}^{f}$ parameter of the radiation transfer model of PT11, we are in a position to directly and independently test the physical consistency and predictive power of Equation (5) using any observable effect which is predicted to be a function of $\tau_{B}^{f}$ by the PT11 model. To this end, we analyze here two quantities which are dependent on the amount and distribution of dust in galaxies, using a much bigger (and largely disjunct in terms of FIR detections) sample than used for the calibration of the $\tau_{B}^{f}-\mu_{*}$ relation, i.e., the OPTICAL sample. These quantities are the inclination dependence of attenuation of NUV emission from late-type galaxies (considered in Section 5.2) and the 
scatter about the well-known scaling relation between the specific SFR $\psi_{*}$ and the stellar mass $M_{*}$ (considered in Section 5.3). We will show that even though the $\tau_{B}^{f}-\mu_{*}$ relation is calibrated on a very limited portion of the overall population of galaxies it is applicable to the general population of spiral galaxies with $7.6<\log \left(\mu_{*}\right)<9.0$.

\subsection{Deriving Attenuation Corrections}

The radiation transfer model presented in Tuffs et al. (2004) and PT11 allows the inclination-dependent attenuation of a spiral galaxy to be calculated for known values of $\tau_{B}^{f}$, disk inclination $i_{d}$, escape fraction $F$, and bulge-to-disk-ratio $B / D$ using Equations (17) and (18) from Tuffs et al. (2004) and the model predictions of attenuation coefficients tabulated in PT11. ${ }^{28}$ For the UV the value of $B / D$ is of negligible importance as the UV emission is almost entirely produced in the disk, even for early-type spirals, and we have assumed $F=0.41$ throughout as calibrated in PT11. Values of $i_{d}$ and $\tau_{B}^{f}$ leading to an object-by-object estimate of attenuation are found as in the following.

\subsubsection{Deriving Inclinations}

The OPTICAL galaxy inclinations are calculated from the $r$-band single Sérsic fit axis ratios of Kelvin et al. (2012) as $i_{d}=\arccos \left((b / a)_{\mathrm{ss}}\right)$, where $(b / a)_{\mathrm{ss}}$ represents the single Sérsic axis ratio in the $r$ band. These inclinations are then corrected for the effects of finite disk thickness as detailed in Section 3 of Driver et al. (2007), with an assumed intrinsic ratio of scale height to semi-major axis of 0.12 .

\subsubsection{Calculating Attenuation-corrected Magnitudes}

Using the cataloged stellar masses (Taylor et al. 2011) and the measured values of $\theta_{e, r}$ (Kelvin et al. 2012) together with Equation (5), we estimate the values of $\tau_{B}^{f}$ for the entire OPTICAL sample. We then correct the NUV absolute magnitudes using the radiation transfer model (Tuffs et al. 2004; utilizing the aforementioned tables of attenuation coefficients in PT11) together with the disk inclination $i_{d}$ and $\tau_{B}^{f}$.

\subsection{The Attenuation-Inclination Relation in the NUV}

Previous work (e.g., Tuffs et al. 2004; Driver et al. 2007; Masters et al. 2010) has predicted and shown that the attenuation of UV/optical emission in spiral galaxies is a strong function of inclination, with this effect being particularly pronounced at shorter wavelengths, thus severely influencing, for example, UV-based tracers of SF. This attenuation-inclination relation implies that the median observed absolute magnitude of members of a given late-type galaxy population should increase as a function of inclination.

Here, given measurements of the inclinations, we use the attenuation-inclination relation to test the predictive power and physical consistency of Equation (5) by calculating the intrinsic absolute NUV magnitudes $M_{\mathrm{NUV}}$, corrected for attenuation as detailed in Section 5.1. On an object-by-object basis the values of $M_{\mathrm{NUV}}$ will display scatter, at the very least due to an intrinsic spread in the galaxies' physical quantities. However, the median of an optically selected sample should no longer

\footnotetext{
28 The requisite data specifying attenuation as a function of different wavelengths are available in electronic form at the CDS via anonymous ftp to cdsarc.u-strasbg.fr (130.79.128.5) or via http://cdsarc.u-strasbg.fr/viz-bin/qcat?J/A+A/527/A109.
}

display an inclination dependence after correction, if the transfer of UV radiation in galaxies is adequately described by the PT11 model, and the $\tau_{B}^{f}-\mu_{*}$ correlation given by Equation (5) is representative of the late-type galaxy population as a whole (in the according range of $\mu_{*}$ ). In particular, given the bias toward massive galaxies in the $O P T I C A L+F I R$, the applicability of Equation (5) to the galaxy population as a whole is by no means obvious.

Figure 4 shows the distributions of corrected and uncorrected absolute NUV magnitude $M_{\mathrm{NUV}}$ as a function of inclination given as $1-\cos \left(i_{d}\right)$, for two subsamples of OPTICAL defined by distinct ranges of $\mu_{*}$, thus corresponding to very different mean values of $\tau_{B}^{f}$. The samples are drawn from the range of observed stellar mass surface density $\mu_{*}$ for which Equation (5) is applicable $\left(7.6<\log \left(\mu_{*}\right)<9.0\right.$; see Section 4.3 and Figure 3), and cover the complete range of available galaxy stellar masses. The ranges in $\mu_{*}$ have been chosen to ensure that the samples are not affected by biases due to noise scattering sources into or out of the range in $\mu_{*}$ for which Equation (5) has been calibrated. We find a median value of $\tau_{B \text {,int }}^{f}=2.26$ for the galaxy sample with $7.8<\log \left(\mu_{*}\right)<8.3$ and a value of $\tau_{B}^{f}=7.04$ for the sample with $8.3<\log \left(\mu_{*}\right)<8.8$. The median value for both samples combined is $\tau_{B}^{f}=3.10$, while that for the entire $O P T I C A L$ sample with $7.6 \leqslant \log \left(\mu_{*}\right) \leqslant 9.0$, the range for which Equation (5) has been calibrated, is $\tau_{B}^{f}=2.42$.

As can be clearly seen the uncorrected samples (red points in Figure 4) display a clear inclination-dependent dimming of their magnitudes, with the median magnitude increasing (i.e., dimming) from the face-on case $\left(1-\cos \left(i_{d}\right)=0\right)$ to the edge-on case $\left(1-\cos \left(i_{d}\right)=1\right)$. In both ranges of $\mu_{*}$, the attenuation-corrected values of $M_{\mathrm{NUV}}$, derived as described in Section 5.1.2, are shown in blue. The corrected values of $M_{\mathrm{NUV}}$ no longer display a dependence on inclination, indicating that the correlation found using the OPTICAL+FIR sample is consistent with the independent observable presented by the attenuation-inclination relation, and with the radiation transfer model of PT11. This is also consistent with the supposition that the bias toward massive/bright sources in the OPTICAL+FIR sample, discussed in Section 4.3 and shown in Figure 3, ${ }^{29}$ does not affect the correlation's applicability to the much larger OPTICAL sample.

These conclusions are reinforced on a quantitative level by the agreement between the observed median distribution of the uncorrected samples and the predicted inclination dependence of a fiducial galaxy with $\tau_{B}^{f}$ corresponding to the median of the sample, and $M_{\mathrm{NUV}}$ corresponding to the median of the corrected bin-wise median $M_{\mathrm{NUV}}$, depicted by the dash-dotted line in Figure 4. In addition to the predicted dependence of attenuation on inclination, the difference in gradient of the attenuation as a function of inclination predicted by PT11 for $\tau_{B}^{f}=2.26$ (corresponding to the range of $7.8<\log \left(\mu_{*}\right)<8.3$ ) and $\tau_{B}^{f}=7.04$ (corresponding to the range of $8.3<\log \left(\mu_{*}\right)<8.8$ ) is also shown in the data.

A large uncertainty in quantitative interpretations of the attenuation-inclination relation such as these arises from the difficulty of correctly classifying edge-on sources due to their intrinsic thickness and bulge component. This may cause these

\footnotetext{
29 The bias of the $O P T I C A L+F I R$ sample toward bright sources is also visible in Figure 4 where the uncorrected (green) and corrected (gray) values of $M_{\mathrm{NUV}}$ for the galaxies in the $O P T I C A L+F I R$ sample in the appropriate range in $\mu_{*}$ are overplotted and predominantly lie at the bright edge of the distribution.
} 


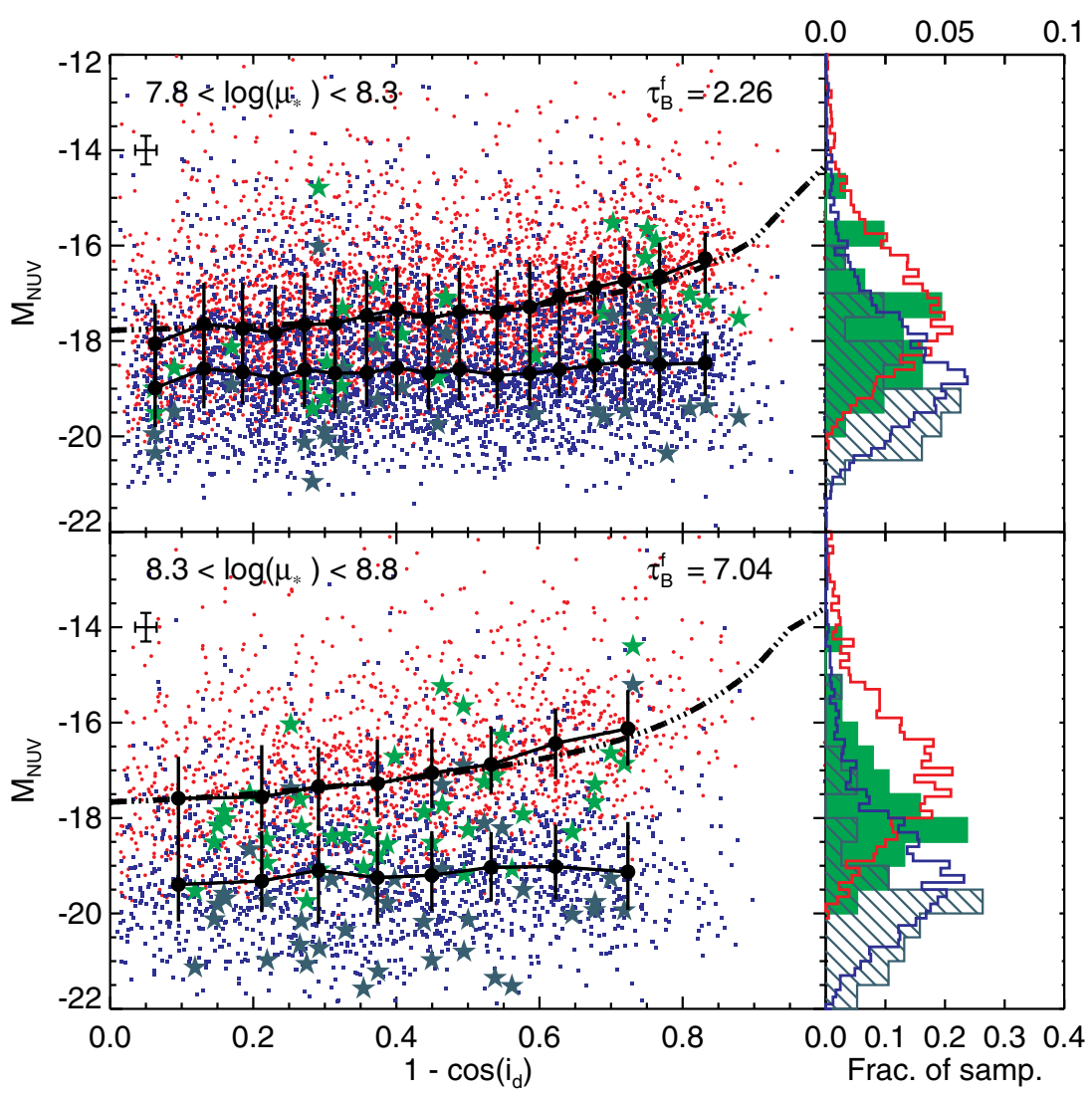

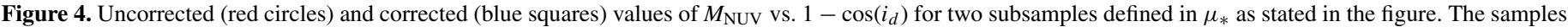

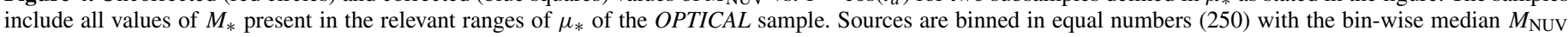

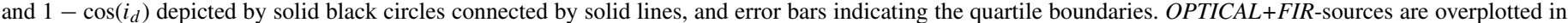

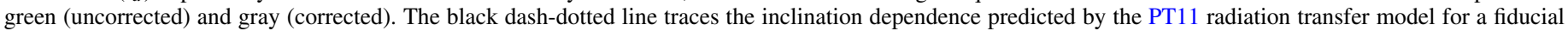

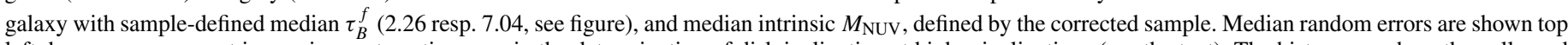

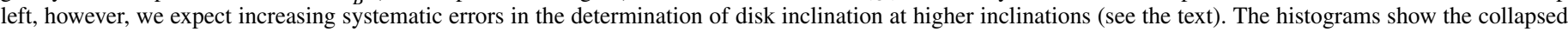

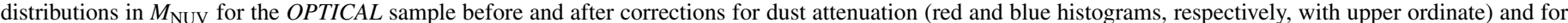
the $O P T I C A L+F I R$ sample also before and after correction (green and blue hatched histograms, respectively, with lower ordinate).

sources to be shifted toward lower values of inclination or to be absent from the sample. Indeed there is a hint that at high inclinations the sample may be slightly biased against low mass galaxies and that dust-rich spirals in general may appear very red at these inclinations leading to a possible misclassification as ellipticals and a bias against edge-on systems. Nevertheless we believe that our results are not affected by strong, inclinationdependent, selection effects, as the samples for both ranges of $\mu_{*}$ are essentially flat in $1-\cos \left(i_{d}\right)$. Furthermore, the distribution of $K$-band absolute magnitudes $M_{K}$ (which are almost free of dust attenuation) shows no inclination dependence indicative of the presence of a strong selection bias.

Both subsamples defined in $\mu_{*}$ display considerable scatter in $M_{\mathrm{NUV}}$ (after correction for attenuation), with the average interquartile range being 1.6, respectively, $1.7 \mathrm{mag}$. This scatter is much larger than can be accounted for by the scatter in $\tau_{B}^{f}$ shown in Figure 2. The range of scatter in $M_{\mathrm{NUV}}$ attributable to the scatter in $\tau_{B}^{f}$, approximated by $\Delta_{s, 0.5} \log \left(\tau_{B}^{f}\right)$ as quoted in Section 4.3 can only account for a range of 0.68 , respectively, $0.9 \mathrm{mag}$ for the ranges $7.8<\log \left(\mu_{*}\right)<8.3$ and $8.3<\log \left(\mu_{*}\right)<8.8$, respectively, even in the edgeon case. Additionally, the interquartile ranges do not display inclination dependence, as would be expected if the scatter were predominantly due to object-by-object variations in the dust opacity. Thus, the sample scatter appears to be dominated by the intrinsic scatter in $M_{\mathrm{NUV}}$. The histograms of $M_{\mathrm{NUV}}$ in Figure 4 clearly show that the corrected sample is more peaked and symmetrical with respect to the uncorrected sample, and that the large shoulder at fainter NUV magnitudes, a product of the inclination dependence, is largely removed after correction. This is the case both for the optically and FIR-selected samples, while the remaining breadth of the distribution (especially for the FIR sample) reinforces the conclusion that the scatter in $M_{\mathrm{NUV}}$ is intrinsic. The remaining tail extending to faint NUV magnitudes can most likely be attributed to passive spirals, as presented, e.g., in Rowlands et al. (2011), and to contamination caused by early-type galaxies $(\approx 5 \%-10 \%)$.

Overall, we conclude that the inclination-dependent dimming of UV emission from galaxies in the complete optical sample can indeed be predicted using the relation between $\mu_{*}$ and $\tau_{B}^{f}$ calibrated on the subset of sources detected in the FIR. The consistency of the correlation with the PT11 model also lends confidence to the supposition that the considerable shift in median magnitude due to the inclination independent component of the attenuation $(\approx 0.9$ and $\approx 1.8 \mathrm{mag}$, as predicted for galaxies seen face-on in the ranges of $7.8<$ $\log \left(\mu_{*}\right)<8.3$ and $8.3<\log \left(\mu_{*}\right)<8.8$, respectively) is also correct, as this is self-consistently derived together with the inclination-dependent component. This is investigated further in Section 5.3. 


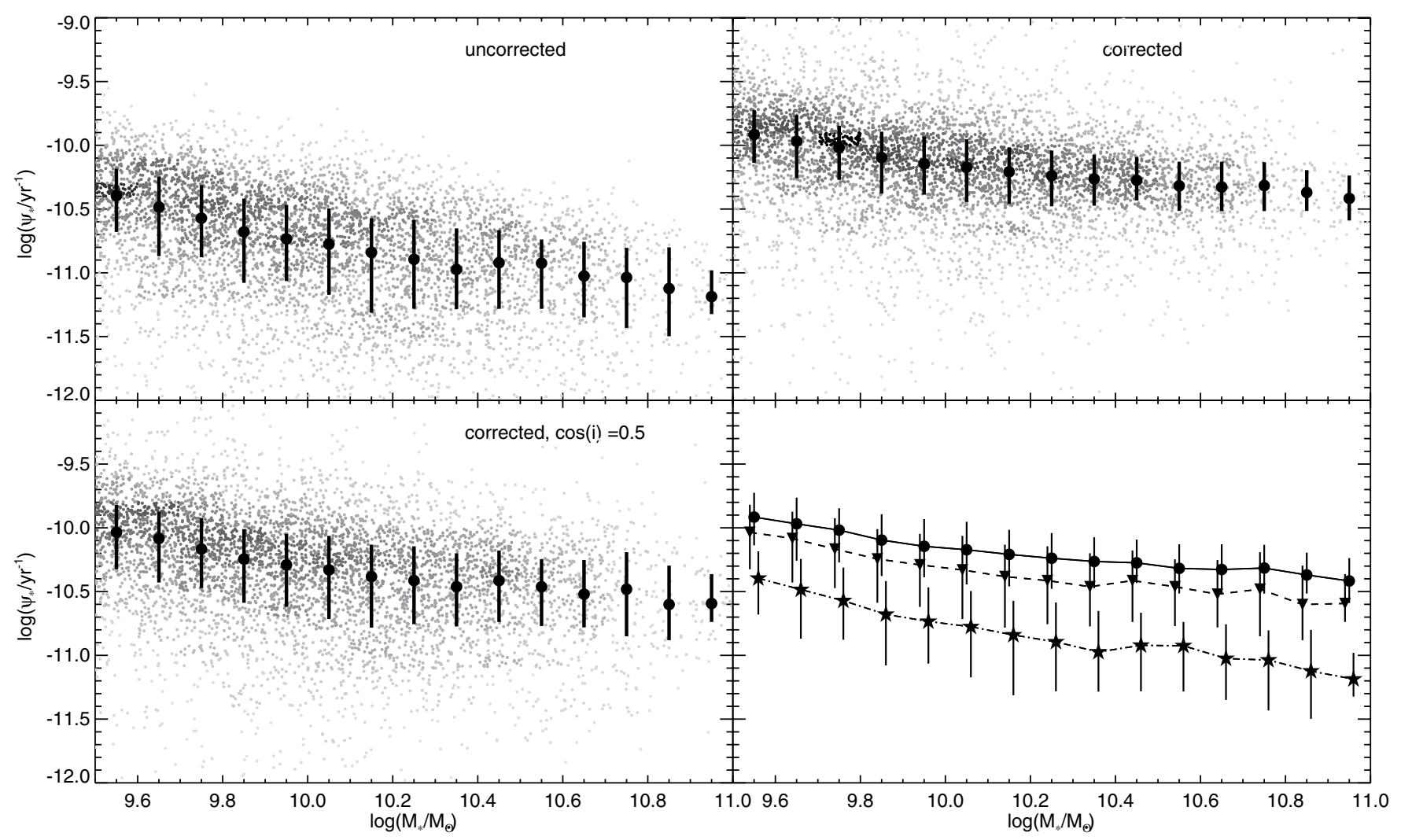

Figure 5. Specific star formation rate $\psi_{*}$ as a function of stellar mass $M_{*}$ for a subsample of the $O P T I C A L$ sample with $7.6 \leqslant \log \left(\mu_{*}\right) \leqslant 9.0$ and $M_{*}>10^{9.5} M_{\odot}$. The relation is shown before correction for attenuation by dust (top left panel), after the full inclination-dependent correction, described in Section 5.1, using the PT11 model in conjunction with the $\tau_{B}^{f}-\mu_{*}$ relation (top right panel), and after a partial correction using the procedure of Section 5.1 but artificially setting a uniform inclination $i_{d}$ with $\cos \left(i_{d}\right)=0.5$ for all galaxies (lower left panel). The sources are binned in 15 bins of equal size in $M_{*}$, with the median depicted by a filled circle, and the bars showing the interquartile range. The scatter in the relation due to the scatter in the NUV is reduced from the uncorrected to the fully corrected case. The intrinsic values of $\psi_{*}$ are shifted upward w.r.t. the uncorrected values. The linear gray scale shows the number density of sources at that position, with the same scale having been applied to all samples. The median values and interquartile ranges are shown together in the bottom right panel. The uncorrected values are depicted by stars and a dash-dotted line, the values corrected at a fixed inclination of $\cos (i)=0.5$ are shown as inverted triangles and a dashed line, and the fully corrected values are shown as circles and a solid line. The bin centers have been offset by 0.01 in $\log \left(M_{*}\right)$ for improved legibility.

\subsection{The Scatter in the Specific Star Formation Rate versus Stellar Mass Relation}

Although we have shown that the $\tau_{B}^{f}-\mu_{*}$ relation in combination with PT11 is effective at predicting the inclinationdependent component of attenuation it is still important to gain a quantitative measure of the efficacy of this technique in predicting the face-on component of the attenuation, which is not so directly probed by the analysis of the attenuation-inclination relation in Section 5.2. Here, we seek to achieve this by utilizing a fundamental scaling relation between physical quantities derived from UV/optical emission properties of galaxies where the intrinsic scatter between the physical quantities is sufficiently small as to be exceeded by the scatter in the observed quantities induced by dust attenuation.

A particularly convenient scaling relation for this analysis is the well-known relation between specific SFR, $\psi_{*}$, and stellar mass, $M_{*}$, since, when derived from NUV magnitudes, the values of SFR used to construct $\psi_{*}$ will be strongly dependent on the efficacy of the method used to correct for attenuation, whereas, as shown by Taylor et al. (2011) and discussed in Section 4, the values of $M_{*}$ are much less affected by dust. Here, we convert from de-attenuated values of $M_{\mathrm{NUV}}$ to $\psi_{*}$ using the conversion given in Kennicutt (1998) scaled from a Salpeter (1955) IMF to a Chabrier (2003) IMF as in Treyer et al. (2007). We note that the exact choice of conversion factor has no bearing on the analysis.
In Figure 5, the $\psi_{*}$ versus $M_{*}$ relation is plotted for the OPTICAL sample limited to $7.6 \leqslant \log \left(\mu_{*}\right) \leqslant 9.0$ and $M_{*}>$ $10^{9.5} M_{\odot}$ (following the range of applicability of the $\tau_{B}^{f}-\mu_{*}$ relation given in Section 4). To differentiate between the effects of the corrections for the face-on and inclination-dependent components of attenuation, we plot the relation three times: with no attenuation corrections (top left), with attenuation corrections as detailed in Section 5.1, but with all inclinations artificially set to the median inclination of the sample (bottom left), and with the corresponding full inclination-dependent corrections (top right). The expected trend of decreasing $\psi_{*}$ as a function of $M_{*}$ is seen in all three panels. Comparison of the top left and top right panels shows, however, that the application of the inclination-dependent attenuation corrections, in addition to inducing a overall systematic shift by a factor of 0.6 dex in $\psi_{*}$, reduces the scatter in the relation, from 0.63 dex in the uncorrected relation to 0.43 dex in the corrected relation. ${ }^{30}$

This suggests a substantial predictive power, both of the $\tau_{B}^{f}-\mu_{*}$ relation and the PT11 model, since we have applied an object-specific and large multiplicative correction to the NUV fluxes (by factors ranging from 2.5 to 6.3 interquartile with a median correction of 3.8), yet have nevertheless succeeded in markedly reducing the logarithmic scatter in the $\psi_{*}$ versus

\footnotetext{
30 All measurements of scatter were calculated as the difference between the quartiles of the distribution in $\psi_{*}$, averaged over 15 equal sized bins in $M_{*}$, and weighted by the number of galaxies in each bin.
} 
$M_{*}$ relation. ${ }^{31}$ Furthermore, comparison of the scatter in the partially corrected relation in the bottom left panel (0.57 dex) with the 0.63 dex scatter in the uncorrected relation in the top left panel shows that the total reduction in scatter is due not only to the correction of the inclination-dependent component of the correction, but also due to the correction of the face-on component of the correction. This is a strong indication that the zero point of the attenuation corrections (i.e., the face-on attenuation predicted by the PT11 model, which is the major contributor to the total attenuation) cannot be strongly in error. If the face-on component of the attenuation would have been independent of the stellar mass surface density, the large range of predicted face-on optical depths at a fixed stellar mass would have increased the scatter, rather than have diminished it.

We note that the intrinsic scatter of 0.43 dex (interquartile) in the corrected relation of Figure 5 (top right panel) will have substantial contributions from random errors. Major sources of this random uncertainty probably arise from measurement uncertainties in the determination of disk surface areas as well as from the estimates of galaxy stellar mass $(\sim 0.1 \mathrm{dex})$. In addition, we recall that the galaxy sample will be contaminated at the 5\%-10\% level by misclassified spheroids. Furthermore we cannot rule out that there is some significant intrinsic scatter in the $\tau_{B}^{f}-\mu_{*}$ relation which would also induce a component of scatter in the corrected $\psi_{*}-M_{*}$ relation. All this suggests that the intrinsic scatter in the $\psi_{*}$ versus $M_{*}$ relation for spiral galaxies may be very low.

\subsection{Implications for the Distribution and Optical Properties of Grains in Galaxy Disks}

The success of the $\tau_{B}^{f}-\mu_{*}$ relation in combination with the PT11 model in predicting both the face-on and inclinationdependent component of the attenuation in spiral galaxies has implications both for the spatial distribution of grains in galaxian disks as well as for the optical properties of these grains.

First, the quantitative consistency between the measured dust surface density and the inclination-dependent attenuation of stellar light in disk galaxies, as predicted by PT11, is consistent with most of the dust in disks being distributed in structures sufficiently large to have a substantial projected surface filling factor. Furthermore, recalling that the $\tau_{B}^{f}-\mu_{*}$ relation is calibrated using measurements of the total submm flux, i.e., tracing the total mass of dust in galaxies, the reduction in scatter about the $\psi_{*}-M_{*}$ relation induced by the application of PT11 points qualitatively toward most of the mass of dust in spiral galaxies (as traced in the submm) being distributed in diffuse, translucent structures, with a large fraction of the grains being exposed to UV in the diffuse interstellar radiation field as assumed by the PT11 model.

In order to make this statement more quantitative, we have in Figure 6 plotted the mean interquartile range in the $\psi_{*}-M_{*}$ relation as a function of attenuation corrections based on an effective value of the dust opacity parameterized by a multiplicative scalar value $\chi$ as $\chi \tau_{B}^{f}$. If, contrary to the model of PT11 in which $\gtrsim 85 \%$ of the total dust mass is diffusely distributed, a large fraction of the dust mass measured in the submm were contained in compact, highly self-shielded regions, not exposed to the diffuse interstellar UV radiation field, the

\footnotetext{
31 As shown from the analysis of the multivariate relation between $\tau_{B}^{f}, M_{*}$, and $\mu_{*}$ in Section 4 the spread in face-on optical depth at a given $M_{*}$ arises from the large spread in disk radii for galaxies of a given $M_{*}$, in conjunction with the close-to-linear $M_{d}$ versus $M_{*}$ correlation.
}

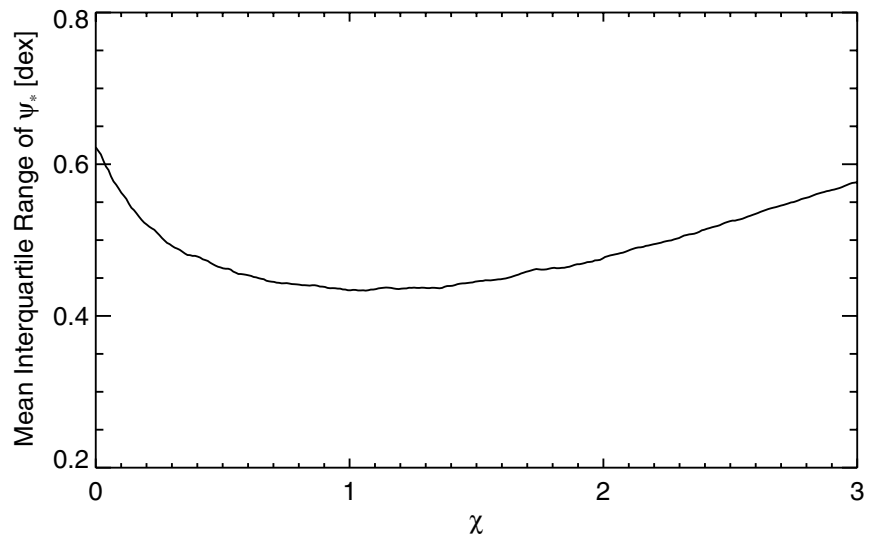

Figure 6. Weighted mean interquartile range of $\psi_{*}$ as a function of $M_{*}$ derived for fractions $\chi \cdot \tau_{B}^{f}$ of $\tau_{B}^{f}$ sampled in steps of 0.01 . The minimum value of 0.43 is attained for $\chi=1.06$, however, it is not significantly distinguishable from that of $\chi=0.95, \ldots, 1.1$.

minimum in scatter about the $\psi_{*}-M_{*}$ relation should be attained for a relatively small value of $\chi$ (i.e., $\chi \ll 1$ ). Instead we find that the minimum scatter is attained for $\chi=1.06$, but that a range of $\chi \approx 0.95-1.1$ is not significantly distinguishable. This implies that, consistent with the PT11 model, a fraction of $\gtrsim 85 \%$ of the total dust mass is distributed in diffuse, translucent structures. A more detailed analysis of the dependence of scatter in scaling relations, though beyond the scope of this paper, could in principle be used to fine tune model assumptions about the fraction of dust in clumps which are heavily self-shielded to UV light in disks of spiral galaxies, and thereby further improve estimates of the absolute level of $\psi_{*}$ in the relation as well as the intrinsic scatter of the physical quantities.

An analysis of the type performed here also has the potential to empirically constrain the ratio between grain emissivities in the submm and UV/optical range. This arises because, while the estimates of $\tau_{b}^{f}$ on which the $\tau_{B}^{f}-\mu_{*}$ relation is based are directly proportional to the dust emission coefficient in the FIR/submm, the amplitude of the attenuation corrections depends upon the dust emission coefficient at (in this case) UV wavelengths. Specifically, the demonstrated ability to correct for the inclination-dependent and face-on components of attenuation using Equation (5), which was derived and calibrated using the FIR, is consistent with the ratio of the UV/optical and submm grain absorption coefficients being as described in the model of Weingartner \& Draine (2001).

\section{DISCUSSION}

\subsection{Dust Production in Spiral Galaxies}

As already noted in Section 4, the almost linear relation between the opacity of a galaxy disk, $\tau_{B}^{f}$, and the surface density of stellar mass, $\mu_{*}$, appears to arise mainly from a roughly linear relation between the extrinsic quantities of total stellar mass, $M_{*}$, and total dust mass, $M_{d}$. Although only established directly for galaxies with available FIR/submm measurements, the applicability of the same $M_{*}-M_{d}$ relation to a statistically much more complete population of optically selected spiral galaxies from the GAMA survey is consistent with our analysis of the attenuation-inclination relation and $\psi_{*}-M_{*}$ relation presented in Section 5.

The physical origin of this link between $M_{*}$ and $M_{d}$ is far from obvious. On the one hand, the stellar mass is dominated 
by old, low mass stars which formed early on in the ca. $10 \mathrm{Gyr}$ lifetime of a typical spiral galaxy. On the other hand, the main known process of injection of dust grains into the ISM is the condensation of metals in the atmospheres of asymptotic giant branch (AGB) stars on timescales of $\sim 2 \pm 1 \times 10^{9} \mathrm{yr}$ (Dwek \& Scalo 1980; McKee 1989; Morgan \& Edmunds 2003; Ferrarotti \& Gail 2006; Zhukovska et al. 2008; Gail et al. 2009; Jones \& Nuth 2011) ${ }^{32}$ much shorter than the ages of spiral galaxies. Moreover, detailed modeling of the life cycle of refractory grains in the solar neighborhood (e.g., Jones et al. 1996; Jones \& Nuth 2011) predicts that grains in the Milky Way are destroyed by supernova (SN) shocks in the tenuous ISM on timescales of $\sim 10^{8} \mathrm{yr}$, much shorter than the timescale for the injection of dust from AGB stars, requiring that almost all observed refractory dust in the diffuse ISM must have been (re-)formed in situ soon after its destruction. This picture of rapid destruction and formation in the ISM is, however, difficult to reconcile with key physical and chemical properties of presolar grains as found in meteorites, most notably the segregation into separate populations of silicate and carbonaceous grains with a high abundance of minerals similar to those known to be produced in stellar sources. As discussed in detail by Jones \& Nuth (2011), one is consequently confronted with a conundrum: either the grain destruction rates in the ISM have been grossly overestimated, allowing most refractory grains in the ISM to have an origin in AGB stars, or, alternatively, an as yet unidentified but very efficient mechanism exists that can convert gaseous metals in a low temperature and low pressure ISM into solid particles with the observed physical and chemical characteristics of interstellar grains.

The close-to-linear relation between $M_{d}$ and $M_{*}$ underlying the $\tau_{B}^{f}-\mu_{*}$ relation naturally favors the existence of a mechanism for efficient growth of refractory dust out of the ISM, since any such mechanism would tap into the full reservoir of metals in the ISM, which are related to the integrated SF over the lifetime of a galaxy. By contrast, if interstellar dust were mainly composed of longer-lived grains injected by AGB stars on timescales of $\sim 2 \pm 1 \times 10^{9} \mathrm{yr}$, one would expect the total mass of dust to be approximately proportional to the SFR multiplied by a residency time, at least for systems with ages larger than the average dust destruction timescale. ${ }^{33}$ Since the residency time should decrease with increasing SFR (since the frequency of destructive $\mathrm{SN}$ shocks should be proportional to the SFR), and the SFR per unit stellar mass is known to decrease as a function of stellar mass, a strongly sublinear dependence between $M_{d}$ and $M_{*}$ would be predicted, even if an increase in the dust yield as a function of metallicity is taken into account. As such, if the origin of dust grains in the ISM were

\footnotetext{
32 Refractory grains have been observed to form in the metal-rich ejecta of core-collapse supernovae, which, alongside type Ia supernovae, have also been postulated to be major sources of interstellar grains. However, with the possible exception of the remnant of SN1987A (Matsuura et al. 2011; Lakićević et al. 2012), FIR/submm measurements of cold unshocked ejecta in the central regions of prototypical young supernova remnants (SNRs) have shown that the ratio of solid state to gas-phase ejecta is modest in comparison to the grain-to-gas ratio in the ISM (e.g., Tuffs et al. 1997; Green et al. 2004; Barlow et al. 2010; Gomez et al. 2011). Given that, to escape the SNR, the condensates must traverse the very shocks postulated to be the main sink for refractory grains in the ISM, it seems unlikely that supernovae are major primary sources of interstellar grains.

33 In the very early evolution of systems, dust mass may increase in parallel with stellar mass simply due to continuous injection of dust into the ISM driven by star formation. Only after the age of the system increases to more than the average dust lifetime can the mass of dust be expected to be proportional to the SFR multiplied by a residency time.
}

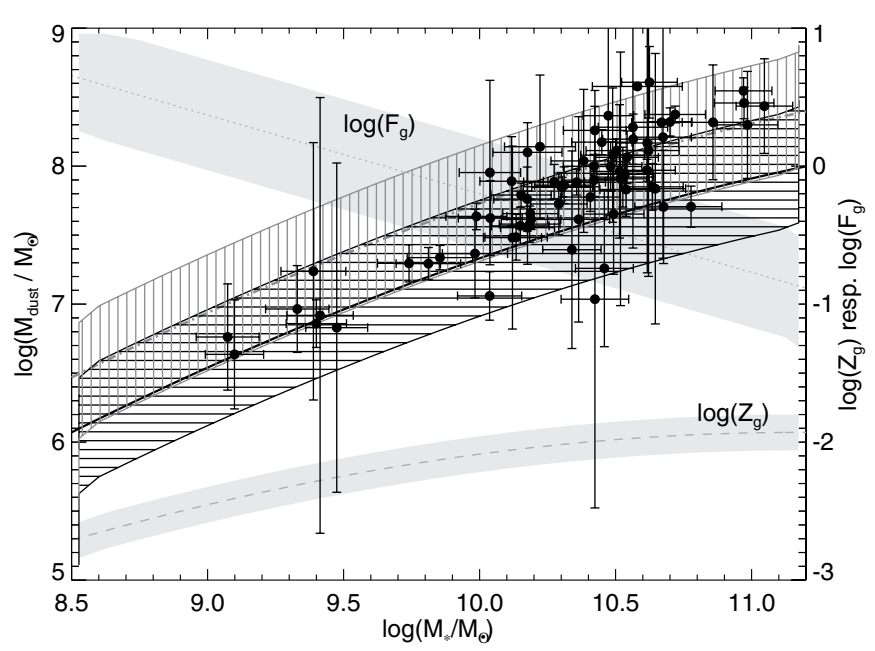

Figure 7. Predicted values of dust mass $M_{\text {dust }}$ as a function of stellar mass $M_{*}$ for an assumed conversion of a fraction $\eta$ of all ISM metals to dust. The mass-metallicity relation (Tremonti et al. 2004; Kewley \& Ellison 2008) converted to gas-phase metallicities, a Chabrier (2003) IMF as in PS11 and the stellar-to-gas mass ratio (PS11) used in deriving the expectations are overplotted as a dashed and dotted lines, respectively, with the shaded areas indicating the range of $1 \sigma$ scatter around the relations. The predicted relation and $1 \sigma$ scatter (derived as sum quadrature) between $M_{\text {dust }}$ and $M_{*}$ is shown for $\eta=0.5$ (solid black line and horizontally striped region) and for $\eta=1$. (dash-dotted gray line and vertically striped region). The diffuse dust masses of the OPTICAL+FIR sample, derived from the values of $\tau_{B}^{f}$ using Equation (1) are overplotted as filled circles with error bars (errors on $M_{\text {dust }}$ take into account errors on $\tau_{B}^{f}$ and $\left.\theta_{e, \mathrm{ss}, r}\right)$.

predominantly stellar, one would expect an at most very weak dependence of dust mass on stellar mass.

If the mechanism for growth of grains out of the ISM implied by the slope of the $\tau_{B}^{f}-\mu_{*}$ relation was sufficiently prompt and efficient it would ubiquitously lead to a high fraction (i.e., of the order unity), $\eta$ of all refractory elements being condensed into grains in the ISM of all spiral galaxies (as also inferred by, e.g., Dwek 1998; Edmunds 2001; Draine 2009). To test whether $\eta$ really does assume a universally high value in the ISM of local universe spiral galaxies, we can make use of the well-established empirical relations linking stellar mass with gas-phase metallicity and gas mass for this galaxy population. Specifically, the product of these relations will yield a relation between total metal mass and $M_{*}$, which, by multiplying the metal mass by a constant value for $\eta$, will predict a relation between total dust mass $M_{d}$ and $M_{*}{ }^{34}$ This relation can then be compared with the observed relation.

Figure 7 shows the predicted relations between $M_{*}$ and $M_{\text {dust }}$ as derived using the mass-metallicity relation for latetype galaxies (Tremonti et al. 2004; Kewley \& Ellison 2008), converted to gas-phase metallicities and a Chabrier (2003) IMF as in Peeples \& Shankar (2011, hereafter PS11), and the gasto-stellar mass ratio from PS11. The relations for $\eta=0.5$ and $\eta=1$ are, respectively, shown by the solid black and dash-dotted gray lines, together with horizontally and vertically striped regions indicating the $1 \sigma$ scatter around the relations.

\footnotetext{
34 The underlying assumption of such a prediction is that all galaxies will have experienced a similar star formation history. Variations in this history can give rise to significant scatter around the relation, in particular, the time at which a large burst of SF occurs may strongly influence the observed dust-to-stellar mass ratio. Nevertheless, as we have endeavored to select a pure sample of normal spiral galaxies, and the specific SFRs obtained for the $O P T I C A L+F I R$ sample do not display bimodality, such an assumption does not appear unreasonable.
} 
It is apparent that the observed trend in $M_{*}$ versus $M_{\text {dust }}$, shown by the overplotted data points from the OPTICAL+FIR galaxies, is indeed quite well predicted by the mass-metallicity and gas mass versus stellar mass relations for constant $\eta$, and that the required value of $\eta$ indeed has to be high. If fact values of between 0.5 and 1 are required for $\eta$, about a factor of two higher than the several tens of percent of ISM metals that are predicted to be present in the form of grains by several detailed physical models such as those by Dwek (1998; $\eta \sim 0.4)$, Edmunds (2001; $\eta \gtrsim 0.4)$, and Galliano et al. (2008; $\eta \sim 0.3$ ). However, as we discuss in Appendix A, it must be born in mind that the measured dust masses plotted in Figure 7 were derived from the FIR/submm observations using a mass absorption coefficient which is actually quite uncertain. In particular, whereas the relative values of the UV and submm grain absorption cross sections of Weingartner \& Draine (2001) used by our radiation transfer analysis connecting the submm emission and UV attenuation characteristics of spiral galaxies have been empirically constrained with respect to hydrogen gas column through measurements of extinction and emission of diffuse dust in the Milky Way, the absolute value of the absorption cross section per unit grain mass $\kappa_{\mathrm{m}}$, needed to deduce the value of $\eta$, is relatively uncertain. As noted by Draine et al. (2007) the value of $\kappa_{\mathrm{m}}$ for the model of Weingartner \& Draine (2001) requires more heavy elements than appear to be available and the mass of dust may be overestimated by a factor of $\sim 1.4 .{ }^{35}$ Such a shift in $\kappa_{\mathrm{m}}$ (while leaving the grain absorption cross sections relative to hydrogen unchanged) would reconcile the majority of the measurements plotted in Figure 7 to an $\eta$ of $\sim 0.5$, given the observed scatter. At the same time, this would preserve the observed quantitative connection between the UV attenuation and the observed surface density of submm emission, as predicted by the PT11 radiation transfer model.

The only way we could envisage maintaining this demonstrated ability to link attenuation of starlight to the observed surface density of submm emission, while avoiding $\eta$ approaching unity and avoiding having to raise $\kappa_{\mathrm{m}}$ for dust in the diffuse ISM from the values given by Weingartner \& Draine (2001), would be to invoke a population of highly self-shielded compact dense clumps as the source for a large fraction of the observed submm emission from spiral galaxies, in conjunction with us having systematically overestimated the intrinsic angular sizes of the disks seen in $r$ band. ${ }^{36}$ While the present accuracy of measurement of intrinsic disk sizes, as outlined in Appendix B, may not completely rule out such a scenario, recent high angular resolution submm imaging of the galactic plane of the Milky Way by the Herschel Space Observatory, sensitive to emission on all angular scales, clearly show that the vast majority of submm

\footnotetext{
35 This is also reflected by the fact that determinations of $\kappa_{\mathrm{m}}$ based on metal abundance as an input constraint predict higher submm grain emissivities than those in the Weingartner \& Draine (2001) dust model. For example, the emissivity model used by Dunne et al. (2011) is partly based on the analysis of James et al. (2002), who, by assuming that $45.6 \%$ of all metals are converted to dust, derived a dust absorption coefficient per unit mass at a wavelength of $850 \mu \mathrm{m}$ which is $\sim 70 \%$ larger than that of Weingartner \& Draine (2001).

36 This would reduce the mass of dust needed to explain the observed submm fluxes, since the dust in self-shielded clumps, while no longer able to efficiently participate in the attenuation of light from stellar populations not spatially correlated with the clumps, might be expected to have a much higher value of $\kappa_{\mathrm{m}}$ in the submm, due to the formation of ice mantles in such environments. The corresponding reduction in the mass of dust in the diffuse ISM would then need to be exactly compensated for by the reduction in the inferred intrinsic angular size of the disk, such as to restore the opacity of the disk to the levels needed to predict the attenuation of the starlight (as quantified through the attenuation-inclination relation and the scatter in the $\psi-M_{*}$ relation).
}

photons originate from translucent large-scale structures (e.g., Molinari et al. 2010).

We conclude that the near linearity and high constant of proportionality of the $\tau_{B}^{f}-\mu_{*}$ relation, itself based on a near linear relation between $M_{\text {dust }}$ and $M_{*}$, is indeed in good agreement with a roughly constant and high (but still physical) fraction of all ISM metals being present in the form of grains, and is best understandable in terms of the existence of a ubiquitous and very rapid mechanism for the in situ growth of grains in the gaseous ISM. Based on a joint consideration of measured dependencies of dust mass, gas fraction, and metallicity on stellar mass, our simple analysis provides a direct and modelindependent empirical confirmation of work which has used more sophisticated chemo-dynamical simulations of the dust cycle in local and high- $z$ galaxies applied to dust abundances and gradients to infer a dominant in situ source of interstellar grains both in local, normal galaxies and high- $z$ starbursts (e.g., Dwek 1998; Calura et al. 2008; Draine 2009; Michałowski et al. 2010; Dunne et al. 2011; Dwek \& Cherchneff 2011; Dwek et al. 2011; Inoue 2011; Mattson et al. 2012; Valiante et al. 2011). Since we make no assumptions about the stellar populations other than that of the current injection rate of stardust being proportional to the recent SF rate, our conclusion that stardust is a minor constituent of dust in spiral galaxies holds even if the IMF for stars were to be top heavy, which has been suggested (e.g., by Dunne et al. 2011) as a possible way of alleviating the need for grain growth in the ISM.

Moreover, the ability of the $\tau_{B}^{f}-\mu_{*}$ relation to predict the NUV attenuation-inclination relation suggests that the majority of grains are exposed to non-ionizing UV light in the diffuse interstellar radiation field, so are refractory in nature ${ }^{37}$ and reside in the diffuse ISM. As a consequence, our results not only require a very efficient grain-formation mechanism, but also require that this mechanism pertains to the formation of refractory grains, rather than merely to the condensation of ices in highly self-shielded regions.

The nature of the mechanism for forming refractory grains in the ISM is completely open. In their comprehensive analysis of the evolution of the interstellar dust population in the solar neighborhood in the Milky Way, based on a one zone chemical evolution model accounting for the growth of individual species, Zhukovska et al. (2008) conclude that the interstellar dust population is dominated by refractory grains grown by accretion of gas-phase metals in dense molecular clouds, with stardust (in their model from both from AGB stars and from $\mathrm{SNe}$ ) constituting only a minor fraction. This result is consistent with our conclusions, independently inferred from the $\tau_{B}^{f}-\mu_{*}$ relation for spiral galaxies, but only provided a mechanism exists to propagate the refractory grains from the clouds into the diffuse ISM on timescales shorter than the timescale for grain destruction in the diffuse ISM. Alternatively, Draine (2009) has proposed that refractory grains can grow in diffuse interstellar clouds, in the presence of UV radiation. This would seem to be more easy to reconcile with our result that the bulk of all grains must reside in translucent structures illuminated by UV, as it would bypass the need for a rapid propagation mechanism.

In general, the $\tau_{B}^{f}-\mu_{*}$ relation may be useful as a diagnostic tool to investigate the universality and nature of the in situ grain-formation mechanism. In particular, although our

\footnotetext{
37 Volatiles in the form of ices will almost instantaneously return to the gas phase through photodesorption if exposed to UV in the diffuse interstellar radiation field.
} 


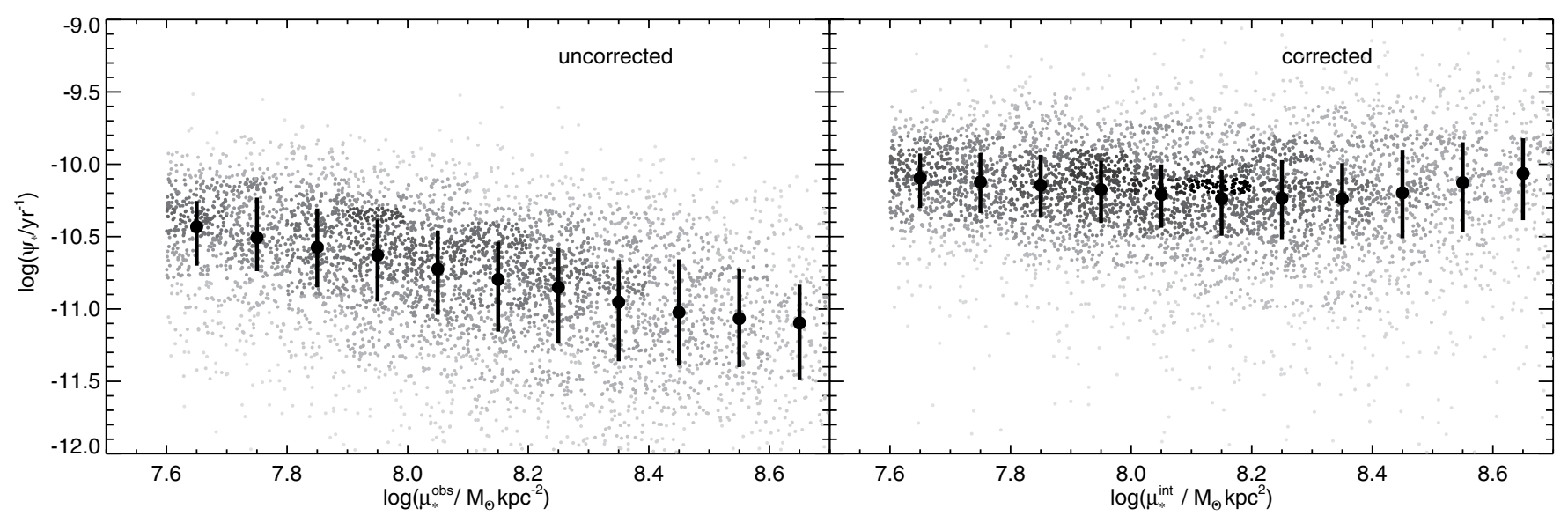

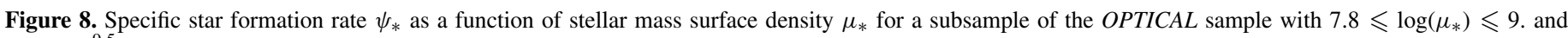

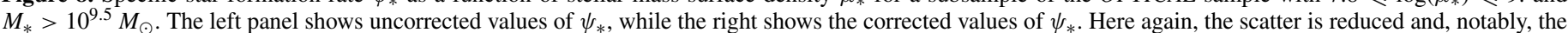

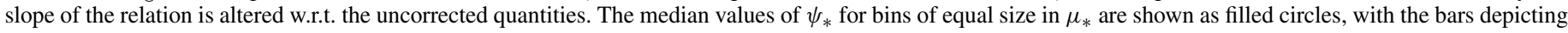

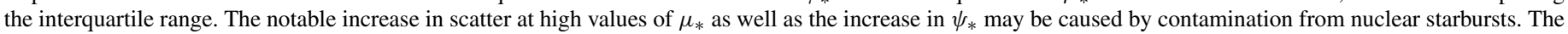
linear gray scale shows the number density of sources at that position, with the same scale having been applied in both panels.

analysis favors grain condensation from the ISM as the main grain injection mechanism, our present statistics cannot rule out that a significant minority of the grains have a stellar origin. Jones \& Nuth (2011) emphasize that there is a considerable uncertainty in theoretical predictions for grain lifetimes, so that, while there is a strong requirement for carbonaceous grains to be rapidly recycled in the ISM, this requirement may be less strong for silicate grains. Analysis of the $\tau_{B}^{f}-\mu_{*}$ relation for larger statistical samples will allow separate relations to be established for spiral galaxies as a function of recent SF history, spiral arm coverage (i.e., lateness) and specific SF rate which may throw more light on this question, particularly if accompanied with data on the strength of the $2200 \AA$ absorption and MIR polycyclic aromatic hydrocarbon emission features, both of which specifically probe carbonaceous particles.

\subsection{The Attenuation of Starlight in Spiral Galaxies}

Having discussed the use of the $\tau_{B}^{f}-\mu_{*}$ relation as a diagnostic of physical processes driving the efficient production of interstellar dust in the disks of spiral galaxies, we return to the main goal of this investigation, namely the use of the $\tau_{B}^{f}-\mu_{*}$ relation, in conjunction with the radiation transfer model of PT11, to correct for the attenuation of stellar light by dust. As described in Section 5, this can be done on a object-to-object basis for large statistical samples of spiral galaxies, using readily available optical photometric properties for each galaxy. Although the predicted attenuations are quite substantial, especially in the UV, our quantitative analysis of the attenuation-inclination and of the scatter in the $\psi_{*}-M_{*}$ relation lends some confidence that the corrections are not largely in error.

As we have already noted in the case of the $\psi_{*}-M_{*}$ relation the scatter in fundamental scaling relations based on UV and optical quantities can be significantly reduced through application of attenuation corrections based on the $\tau_{B}^{f}-\mu_{*}$ relation even when no dust emission data are available. The 0.43 dex interquartile scatter in the $\psi_{*}-M_{*}$ relation after correcting for attenuation already implies a very tight relation between the current and past SF in spiral galaxies in the local universe that would need to be reproduced by any theory of the formation and growth of spiral galaxies.
Although affecting the scatter of the $\psi_{*}$ versus $M_{*}$ relation, the attenuation corrections do not, as already noted, strongly affect the slope of this relation, at least in the range of $M_{*}$ for which the attenuation corrections are presently available through the $\tau_{B}^{f}-\mu_{*}$ relation. This is because, as shown in Section 4, opacities are statistically much more tightly related to stellar mass surface density, rather than to stellar mass, coupled with the fact that spiral galaxies of a given stellar mass exhibit a wide range of disk sizes. However, this situation will no longer apply to scaling relations as a function of the stellar surface mass density, $\mu_{*}$. To illustrate this, we plot in Figure 8 the relation between $\psi_{*}$ and $\mu_{*}$, for the same sample as used for the $\psi_{*}$ versus $M_{*}$ relation, both before and after correction. Remarkably, the slope in the relation between the uncorrected quantities is entirely removed after correction for dust, showing that $\psi_{*}$ is statistically independent of $\mu_{*}$. The scatter in the relation is reduced from 0.62 to 0.49 dex. The latter value is somewhat larger than that for the $\psi_{*}$ versus $M_{*}$ relation, perhaps implying that the $\psi_{*}$ versus $M_{*}$ relation has a smaller intrinsic scatter and is thus the more fundamental relation.

Overall, the fact that disk opacities scale systematically with stellar mass surface density, as opposed to being randomly distributed, may help to explain the preservation of systematic and in some cases surprisingly tight relations between optical or UV tracers of physical quantities, even when these observational tracers are heavily affected by dust attenuation, and may help to explain why many relations were historically discovered only with relatively crude corrections for dust attenuation. Apart from the scaling relations analyzed here, a further relation which would be particularly pertinent to reanalyze would be the Tully-Fisher (TF) relation between luminosity and dynamical mass (Tully \& Fisher 1977), which is even tighter than the $\psi_{*}$ versus $M_{*}$ relation. A similar analysis to that applied to the $\psi_{*}$ versus $M_{*}$ relation here applied to the TF relation could provide a still sharper tool for statistical analysis of attenuation corrections, as well as potentially improving the accuracy of the TF relation at shorter wavelengths, both as a distance indicator, and as a diagnostic of the formation and evolution of spiral galaxies.

Finally, we re-emphasize that the corrected relations are only for subsets of the galaxy population restricted in $M_{*}$ and $\mu_{*}$ according to the limits of our present calibration of the $\tau_{B}^{f}-\mu_{*}$ 
relation as defined in Section 4. In order to establish attenuationcorrected scaling relations for the whole population of disk galaxies, it is crucial to extend knowledge of FIR/submm emission, and thereby calibration of the $M_{*}$ and $\mu_{*}$ relation, to a representative subset of the entire population of rotationally supported disk galaxies, including dwarf galaxies.

\section{SUMMARY AND OUTLOOK}

We have presented a correlation between the face-on $B$-band dust-opacity $\tau_{B}^{f}$ and the stellar mass surface density $\mu_{*}$ for normal late-type galaxies for a range in $\mu_{*}$ (in units of $M_{\odot} \mathrm{kpc}^{-2}$ ) of $7.6<\log \left(\mu_{*}\right)<9.0$. Using the attenuation-inclination relation for rotationally supported late-type galaxies, we have demonstrated that the values of $\tau_{B}^{f}$ estimated by means of this correlation successfully correct statistical samples of late-type galaxies for dust attenuation. In order to apply the correlation to complete samples of spiral galaxies in terms of $M_{*}$, the present depth of FIR/submm data used to derive the $\tau_{B}^{f}-\mu_{*}$ correlation means such samples need to be limited to $M_{*} \geqslant 10^{9.5} M_{\odot}$. While the selection method employed to select spirals depends on the availability of NUV and optical data, only photometric optical data is needed to estimate the values of $\tau_{B}^{f}$, as the required Sérsic fits are performed on optical ( $r$-band) imaging, and stellar masses have been derived using only optical data. As such this correlation presents a means to obtain attenuation corrections for samples of galaxies on the basis of optical photometric data alone, and is thus applicable to a large range of data sets.

We quantitatively demonstrate the efficacy of attenuation corrections using the $\tau_{B}^{f}-\mu_{*}$ relation in concert with the PT11 model through analysis of the attenuation-inclination relation in the NUV and the $\psi_{*}$ versus $M_{*}$ relation for a large sample of local universe spiral galaxies. Both the inclination-dependent and the face-on components of attenuation are well predicted; for the latter we find that the scatter in the $\psi_{*}$ versus $M_{*}$ relation is minimized for corrections within $10 \%$ of those estimated using the $\tau_{B}^{f}-\mu_{*}$ relation and the PT11 model, consistent with the large majority of dust residing in the diffuse ISM, rather than in opaque clouds. Overall our results are consistent with a general picture of spiral galaxies in which most of the submm emission originates from grains residing in translucent structures exposed to UV in the diffuse interstellar radiation field.

We identify a roughly linear relation between the dust mass and stellar mass of a galaxy for the range of $M_{*}$ probed by our sample to be the dominant driver for the $\tau_{B}^{f}-\mu_{*}$ correlation. Combining this result with known empirical dependencies of the gas-to-stellar mass ratio and metallicity on stellar mass, we make a largely model-independent inference requiring the rapid (re-)formation of dust grains after their destruction in the ISM, as has also been inferred from chemo-dynamical modeling of the dust cycle in the Milky Way and external galaxies. Our requirement that the reformed dust is refractory and largely resides in the diffuse ISM (rather than in dense clouds) will further constrain models of the dust cycle in galaxies. We posit that the $\tau_{B}^{f}-\mu_{*}$ relation applied to statistical samples of late-type galaxies subdivided by, e.g., morphology/lateness and SFR, may provide a diagnostic tool to investigate the applicability to late-type galaxies in general of results pertaining to grain formation derived from studies of the local ISM in the Milky Way.
At the moment, the determination of $\tau_{B}^{f}$ using modified blackbody functions as well as the uncertainty, introduced by the effects of diffuse dust and the bulge-to-disk ratio, on the measured scale lengths of galaxies are major contributors to the uncertainties in the results presented. On the other hand, however, the approach chosen in this paper which gives rise to these uncertainties, also ensures that the result given by Equation (5) is not strongly model dependent, and is straightforward to implement in practical applications. In the future, we will return to this topic using a larger data set and compare these results with such obtained using values of $\tau_{B}^{f}$ and $\theta_{s, d, r}$ stemming from a fully self-consistent radiation transfer modeling approach (cf. PT11). Future work will also focus on extending the range in $\mu_{*}$ (hence also in $M_{*}$ ) over which the relation is applicable using fully self-consistent radiation transfer modeling, two component Sérsic fits (using future higher resolution photometry as the data becomes available from VST KIDS, VISTA VIKING), and a larger data set in the form of the entirety of H-ATLAS data.

GAMA is a joint European-Australasian project based around a spectroscopic campaign using the Anglo-Australian Telescope. The GAMA input catalog is based on data taken from the Sloan Digital Sky Survey and the UKIRT Infrared Deep Sky Survey. Complementary imaging of the GAMA regions is being obtained by a number of independent survey programs including GALEX MIS, VST KIDS, VISTA VIKING, WISE, HerschelATLAS, GMRT, and ASKAP providing UV to radio coverage. GAMA is funded by the STFC (UK), the ARC (Australia), the AAO, and the participating institutions. The GAMA Web site is http://www.gama-survey.org. The Herschel-ATLAS is a project with Herschel, which is an ESA space observatory with science instruments provided by European-led Principal Investigator consortia and with important participation from NASA. The H-ATLAS Web site is http://www.h-atlas.org. Galaxy Evolution Explorer (GALEX) is a NASA Small Explorer, launched in 2003 April. We gratefully acknowledge NASA's support for construction, operation, and science analysis for the GALEX mission, developed in cooperation with the Centre National d'Etudes Spatiales (CNES) of France and the Korean Ministry of Science and Technology. R.J.T. thanks Wolfgang Krätschmer for discussions on the formation and growth of interstellar grains. M.W.G. acknowledges the support of the International Max-Planck Research School on Astronomy and Astrophysics Heidelberg (IMPRS-HD).

\section{APPENDIX A}

\section{THE RELATION BETWEEN DISK OPACITY, FIR/SUBMM FLUX DENSITY, AND DISK SCALE LENGTH IN TERMS OF THE PT11 MODEL}

In the PT11 model, the opacity of the disk of a spiral galaxy is determined by the mass distribution of the diffuse dust component. This is modeled as the sum of two exponential disks and has been constrained by the reproducible trends found in the radiation transfer analysis of the galaxy sample of Xilouris et al. (1999). For such an axisymmetric distribution of diffuse dust, the face-on optical depth $\tau_{v}(r)$ at a given frequency $v$ and a given radial position $r$ is related to the dust surface density $\Sigma(r)$ and the dust spectral emissivity $\kappa_{v}$ as $\tau_{v}(r)=\Sigma(r) \kappa_{v}$. Accordingly, for each disk $i$ in the PT11 model, $\tau_{v, i}(r)$ (the face-on optical depth at frequency $v$ and radial position $r$ of the disk $i$ ) can be 
expressed as

$$
\begin{aligned}
\tau_{v, i}(r) & =\Sigma_{0, i} \kappa_{\text {ref }} f(v) \exp \left(\frac{-r}{r_{s, d, \text { ref }, i}}\right) \\
& =\tau_{0, \text { ref }} f(v) \exp \left(\frac{-r}{r_{s, d, \text { ref }, i}}\right),
\end{aligned}
$$

where $\Sigma_{0, i}$ is the central dust surface density of the disk $i, r_{s, d, \text { ref, } i}$ is the scale length of the disk $i$ at a reference wavelength, $\kappa_{\text {ref }}$ is the dust emissivity at a reference frequency, and $f(v)$ describes the frequency dependence of the dust emissivity given by the Weingartner \& Draine (2001) dust model $(f(v)$ is not analytically known). Clearly, in this model geometry, the value of $\Sigma_{0, i}$ is proportional to the mass of dust in the disk $i$ and inversely proportional to the area of the disk, respectively the scale length squared, i.e.,

$$
\Sigma_{0, i} \propto \frac{M_{\mathrm{dust}}}{r_{s, d, \mathrm{ref}, i}^{2}} .
$$

Thus, with the opacity of the PT11 model consisting of the sum of two such exponential disks, the optical depth at a given wavelength and position can be fully expressed in terms of the central face-on density of dust, respectively, the face-on opacity in a reference band (the $B$ band at $4430 \AA$ for PT11) as

$$
\tau_{B}^{f}=K \frac{M_{\mathrm{dust}}^{\mathrm{diff}}}{r_{s, d, B}^{2}}
$$

where $K=1.0089 \mathrm{pc}^{2} \mathrm{~kg}^{-1}$ is a constant containing the details of the geometry and the dust model of Weingartner \& Draine (2001). Following the PT11 model, the total mass of dust $M_{\text {dust }}$ in a galaxy is given by

$$
M_{\mathrm{dust}}=M_{\mathrm{dust}}^{\mathrm{diff}}+M_{\mathrm{dust}}^{\text {clump }}=(1+\xi) M_{\mathrm{dust}}^{\mathrm{diff}},
$$

where $M_{\text {dust }}^{\text {diff }}$ is the mass of diffusely distributed dust and $M_{\text {dust }}^{\text {clump }}$ is the mass of dust in self-shielded clumps, not partaking in the attenuation of optical emission. PT11 find the mass fraction of these clumps to be low $(\sim 10 \%-15 \%)$. ${ }^{38}$ Given the uncertainties on the measurement of dust masses in comparison to the likely value of $\xi$, $\xi$ may be neglected to obtain:

$$
\tau_{B}^{f}=K \frac{M_{\mathrm{dust}}^{\mathrm{diff}}}{r_{s, d, B}^{2}} \approx K \frac{M_{\mathrm{dust}}}{r_{s, d, B}^{2}},
$$

i.e., Equation (1) in Section 2.

Estimating $\tau_{B}^{f}$ from observable quantities requires several assumptions in order to re-express Equation (A3) in terms of observables. Under the assumption that the dust emission in the FIR, i.e., at wavelengths longward of $100 \mu \mathrm{m}$, can be approximated by a modified Planckian with emissivity $\beta$, the total mass of dust in the galaxy can be expressed as

$$
\begin{aligned}
M_{\text {dust }} & =\frac{L_{v}\left(v_{\mathrm{em}}\right)}{4 \pi \kappa_{v_{\mathrm{cal}}}\left(\frac{v_{\mathrm{em}}}{v_{\mathrm{cal}}}\right)^{\beta} B\left(v_{\mathrm{em}}, T_{0}\right)} \\
& =\frac{S_{v}\left(v_{\mathrm{ob}}\right) D_{\mathrm{L}}^{2}(z) v_{\mathrm{cal}}^{\beta}}{(1+z)^{1+\beta} \kappa_{v_{\mathrm{cal}}} v_{\mathrm{ob}}^{\beta} B\left((1+z) v_{\mathrm{ob}}, T_{0}\right)},
\end{aligned}
$$

\footnotetext{
38 This assumes the emissivity of dust in clumps is the same as that of diffuse dust. As cold self-shielded environments are conducive to the formation of ices with greater emissivity coefficients, this estimate likely represents a upper bound.
}

where $L_{v}\left(v_{\mathrm{em}}\right)$ is the luminosity density at the frequency $v_{\mathrm{em}}$ related to the observed frequency $v_{\mathrm{ob}}$ as $v_{\mathrm{ob}}=(1+z) v_{\mathrm{em}}, S_{\nu}\left(v_{\mathrm{ob}}\right)$ is the observed flux density, $\kappa_{v_{\text {cal }}}$ is the emissivity coefficient at the frequency $v_{\text {cal }}, B(v, T)$ is the Planck function evaluated at frequency $v$ and temperature $T, T_{0}$ is the rest-frame temperature of the source, $z$ is the redshift of the source, and $D_{\mathrm{L}}(z)$ is the source's luminosity distance. Similarly, the physical scale length of the disk $r_{s, d, B}$ can be expressed as an angular size $\theta_{s, d, B}$ as

$$
r_{s, d, B}=\theta_{s, d, B} D_{\mathrm{A}}(z)=\theta_{s, d, B} \frac{D_{\mathrm{L}}(z)}{(1+z)^{2}},
$$

where $D_{\mathrm{A}}(z)$ is the angular diameter distance corresponding to the redshift $z$. Using Equations (A4), (A6), and (A7) Equation (A3) can be expressed as

$$
\begin{aligned}
\tau_{B}^{f} & =\frac{K}{(1+\xi)} \frac{S_{v}\left(v_{\mathrm{ob}}\right) D_{\mathrm{L}}^{2}(z) v_{\mathrm{cal}}^{\beta}}{(1+z)^{1+\beta} \kappa_{v_{\mathrm{cal}}} v_{\mathrm{ob}}^{\beta} B\left((1+z) v_{\mathrm{ob}}, T_{0}\right)} \frac{(1+z)^{4}}{\theta_{s, d, B}^{2} D_{\mathrm{L}}^{2}(z)} \\
& =\frac{K}{(1+\xi) \kappa_{v_{\mathrm{cal}}} v_{\mathrm{cal}}^{-\beta} v_{\mathrm{ob}}^{\beta} \gamma^{2}} \frac{(1+z)^{3-\beta}}{B\left((1+z) v_{\mathrm{ob}}, T_{0}\right)} \frac{S_{v}\left(v_{\mathrm{ob}}\right)}{\theta_{s, d, r}^{2}} \\
& =A \frac{(1+z)^{3-\beta}}{B\left((1+z) v_{\mathrm{ob}}, T_{0}\right)} \frac{S_{\nu}\left(v_{\mathrm{ob}}\right)}{\theta_{s, d, r}^{2}}
\end{aligned}
$$

corresponding to Equation (2) in Section 2, with the fixed geometry of the PT11 model being used to re-express $r_{s, d, B}$ as $r_{s, d, B}=\gamma r_{s, d, r}$ (although we set $\xi=0$ in the work presented here for the purpose of determining dust masses, we have chosen to include the factor $(1+\xi)$ in the derivation presented here for purposes of completeness). Although the approximation of the dust emission from a galaxy by a single temperature modified Planckian is a reasonable assumption at FIR/submmm wavelengths, real galaxies will tend to have a range of components of different temperatures and the temperature derived will correspond to a luminosity weighted average temperature. Furthermore, the emissivity of the dust model of Weingartner \& Draine (2001) is only approximately a modified Planckian with a fixed emissivity $\beta$, and the actual mass fraction of dust in clumps is not known and difficult to constrain, as the emissivity in these regions may vary with respect to that in the diffuse medium. Here, we have attempted to take these effects into account in first order by empirically determining the numerical value of $A$ using the radiation transfer solutions to the Xilouris et al. (1999) galaxy sample, in particular NGC 891. For a known source with $\tau_{B}^{f}=\tau_{B, \text { ref }}^{f}, \theta_{r, s, r, \text { ref }}=\theta_{r, s, r}^{\text {ref }}, S_{\nu}\left(v_{\mathrm{ob}}\right)=S_{v}^{\text {ref }}\left(v_{\mathrm{ob}}\right), z=z_{\mathrm{ref}}$, and $T_{0}=T_{0}^{\text {ref }}$ Equation (A8) can be used to identify $A$ as

$$
A=\tau_{B, \mathrm{ref}}^{f} \frac{\theta_{r, s, r, \mathrm{ref}}^{2}}{S_{\nu}^{\mathrm{ref}}\left(v_{\mathrm{ob}}\right)} \frac{B\left(\left(1+z_{\mathrm{ref}}\right) \nu_{\mathrm{ob}}, T_{0}^{\mathrm{ref}}\right)}{\left(1+z_{\mathrm{ref}}\right)^{3-\beta}} .
$$

From the analysis of the Xilouris et al. (1999) galaxy sample, in particular NGC 891 as presented in PT11, we obtain $A=$ $6.939 \times 10^{-13} \operatorname{arcsec}^{2} \mathrm{~J} \mathrm{Jy}^{-1} \mathrm{~s}^{-1} \mathrm{~Hz}^{-1} \mathrm{~m}^{-2} \mathrm{sr}^{-1}$ using $\lambda_{\mathrm{ob}}=$ $250 \mu \mathrm{m}, \tau_{B, \text { ref }}^{f}=3.5, \theta_{r, s, r, \text { ref }}=116^{\prime \prime}, S_{v}^{\text {ref }}\left(v_{250}\right)=115 \mathrm{Jy}$, and $T_{0}^{\text {ref }}=20.74 \mathrm{~K}$ at a distance of $9.5 \mathrm{Mpc}$. This empirical calibration implicitly takes the mass fraction of clumps as assumed in the PT11 model into account, hence derived dust masses may be expected to be slightly underestimated $(\lesssim 10 \%)$.

Finally, we wish to draw attention to the fact that the grain absorption cross sections in the UV and FIR of the Weingartner \& Draine (2001) model have been empirically constrained with 
respect to the hydrogen gas column through measurements of extinction and emission of diffuse dust in the Milky Way. Thus, the values of opacity are empirically constrained per unit hydrogen column, i.e., $\kappa_{v}=\kappa_{v, \mathrm{H}}$. The derivation of dust masses, as e.g., given above, however, requires the absorption cross sections to be expressed per unit grain mass, i.e., $\kappa_{v}=\kappa_{v, \mathrm{~m}}$. With the conversion unit hydrogen column to unit grain mass being relatively uncertain, the cross sections in the UV and FIR/submm are much more tightly constrained with respect to each other, than their absolute values. For example, as noted in Draine et al. (2007), the value of $\kappa_{v, \mathrm{~m}}$ for the model of Weingartner \& Draine (2001) requires more heavy elements than are expected to be available and may easily overestimate the mass of dust by a factor of $\sim 1.4$. In terms of the analysis presented here, such an overestimate will only affect absolute values such as dust masses, while leaving the predicted attenuations unaffected.

\section{APPENDIX B}

\section{THE RELATION BETWEEN APPARENT AND INTRINSIC SIZES}

Spiral galaxies are fundamentally multi-component systems, consisting, to first order, of a disk and a bulge. Nevertheless, their light profiles are often fitted using single Sérsic profiles, especially in the case of marginally resolved systems. In order to link the observed sizes, i.e., the effective radius, to the intrinsic length scales of the disk and the bulge, multiple factors must be considered. While the ratio between effective radius and scale length for a simple exponential disk is 1.68 , the ratio between the effective radius of a single Sérsic-profile fit to a bulge + disk system and the scale length of the disk component will decrease as the importance of the bulge increases. Conversely, the presence of diffuse dust in a late-type galaxy will influence the measured sizes of these objects if the surface density of diffuse dust possesses a radial gradient. Under these circumstances, the apparent size measured will tend to be larger than the intrinsic size. The severity of this effect depends on both the value of $\tau_{B}^{f}$ and the inclination of the disk $i_{d}$, and is sensitive to the details of the dust geometry in the galaxy. Furthermore, as the degree of attenuation caused by diffuse dust varies as a function of wavelength, the effect will also be wavelength dependent. This effect has been quantitatively predicted for pure disk systems (Möllenhoff et al. 2006; Pastrav et al. 2013), and has been observed in the wavelength dependence of galaxy sizes (e.g., Kelvin et al. 2012; Häußler et al. 2013). A joint consideration of these effects, investigating the combined dependencies of the ratio between the observed single Sérsic effective radius and the scale length of the disk component on wavelength, bulgeto-disk ratio $B / D$, inclination $i_{d}$, and $\tau_{B}^{f}$ has been performed by B. Pastrav et al. (in preparation). Figure 9 shows the $r$-band ratio as a function of inclination for four values of $B / D$ at fixed $\tau_{B}^{f}$ (top) and four values of $\tau_{B}^{f}$ at fixed $B / D$ (bottom). Pastrav et al., have performed this analysis using synthetic images of galaxies created using the same geometry assumed in PT11 and, accordingly, the use of these corrections is entirely consistent with the use of the PT11 radiation transfer model.

$$
\text { B.1. Determining } r_{d, s} \text { and } \tau_{B}^{f}
$$

Equation (2) enables the determination of $\tau_{B}^{f}$ based on the observed FIR flux $S_{250}$ and the angular size corresponding to the disk scale length in the $r$-band $\theta_{s, d, r}$. Taking the corrections

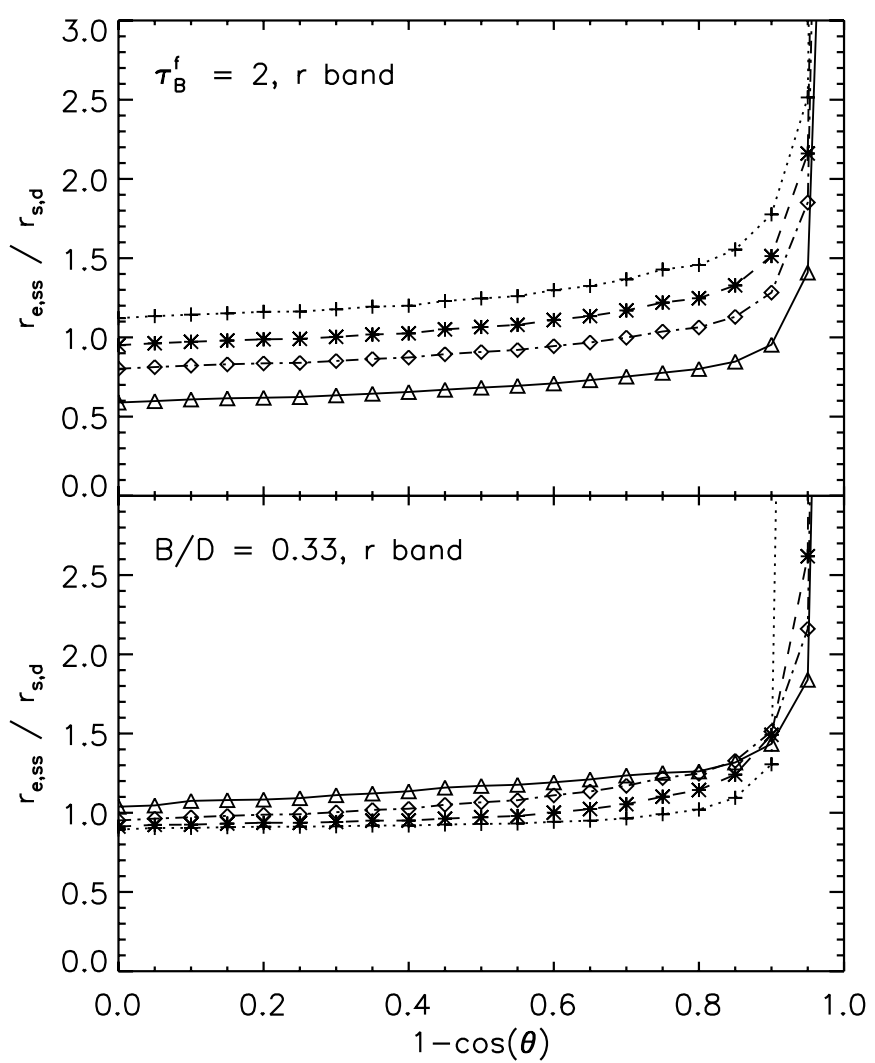

Figure 9. Top: ratio between effective radius observed fitting a single Sérsic profile $\left(r_{e, \mathrm{ss}}\right)$ and the scale length of the disk component $\left(r_{s, d}\right)$ as a function of inclination, for four values of $B / D: 0.25$ (dotted crosses), 0.33 (dashed stars), 0.4 (dash-dotted diamonds), and 0.5 (triple-dash-dotted triangles) observed in the $r$ band. Bottom: ratio between effective radius observed fitting a single Sérsic profile $\left(r_{e}^{\mathrm{ss}}\right)$ and the scale length of the disk component $\left(r_{s}^{d}\right)$ as a function of inclination, for four values of $\tau_{B}^{f}$ at $B / D=0.33$ : 0.5 (dotted crosses), 1.0 (dashed stars), 2.0 (dash-dotted diamonds), and 4.0 (triple-dash-dotted triangles) observed in the $r$ band. Notice the ratio of order unity for values of $B / D$ corresponding to (massive) spiral galaxies, rather than 1.68 as expected for pure disk systems. Data from B. Pastrav et al. (in preparation).

into account, Equation (2) can be expressed as

$$
\tau_{B}^{f} \propto \frac{S_{250}}{\theta_{e, \mathrm{ss}, r}} R\left(\tau_{B}^{f}, i_{d}, B / D\right),
$$

where $R\left(\tau_{B}^{f}, i_{d}, B / D\right)$ is the inverse of the ratio between $r_{e, \mathrm{ss}, r}$ (the physical effective radius in the $r$ band obtained from the single Sérsic fit) and $r_{s, d, r}$ as derived using the ratios of Pastrav et al. These ratios are provided in tabulated form, and we have interpolated the ratios in $\lambda, i_{d}$, and $B / D$ and have fit the $\tau_{B}^{f}$ dependence using a cubic spline. Using this spline, Equation (B1) is solved numerically, obtaining the values of $\tau_{B}^{f}$ and $r_{s, d, r}$ for the galaxy. In determining the values of $\tau_{B}^{f}$ for the $O P T I C A L+F I R$ sample, we have used a value of $B / D=0.33$, representative of the massive spirals in the sample (Graham $\&$ Worley 2008). We caution, however, that the value of $B / D$ is a major source of uncertainty, which will be addressed in future work as and when higher resolution imaging, enabling morphological decompositions of the bulge + disk, becomes available.

\section{REFERENCES}

Abazajian, K. N., Adelman-McCarthy, J. K., \& Agüeros, M. A. 2009, ApJS, 182,543 
Baes, M., Fritz, J., Gadotti, D. A., et al. 2010, A\&A, 518, L39

Baes, M., Verstappen, J., De Looze, I., et al. 2011, ApJS, 196, 22 Baldry, I. K., Robotham, A. S. G., Hill, D. T., et al. 2010, MNRAS, 404, 86 Bamford, S. P., Nichol, R. C., Baldry, I. K., et al. 2009, MNRAS, 393, 1324 Barlow, M. J., Krause, O., Swinyard, B. M., et al. 2010, A\&A, 518, L138 Bendo, G. J., Boselli, A., Dariush, A., et al. 2011, MNRAS, 419, 1833 Bianchi, S. 2008, A\&A, 490, 0461

Bianchi, S., Davies, J. I., \& Alton, P. B. 2000, A\&A, 359, 65

Boselli, A., Eales, S., Cortese, L., et al. 2010, PASP, 122, 261

Bourne, N., Maddox, S. J., Dunne, L., et al. 2012, MNRAS, 412, 3027

Braine, J., Gratier, P., Kramer, C., et al. 2010, A\&A, 518, L69

Bruzual, G., \& Charlot, S. 2003, MNRAS, 344, 1000

Calura, F., Pipino, A., \& Matteucci, F. 2008, A\&A, 479, 669

Calzetti, D. 2001, PASP, 113, 1449

Calzetti, D., Armus, L., Bohlin, R. C., et al. 2000, ApJ, 533, 682

Cardelli, J. A., Clayton, G. C., \& Mathis, J. S. 1989, ApJ, 345, 245

Chabrier, G. 2003, PASP, 115, 763

Cortese, L., Ciesla, L., Boselli, A., et al. 2012, A\&A, 540, 52

da Cunha, E., Charlot, S., \& Elbaz, D. 2008, MNRAS, 388, 1595

Dariush, A., Cortese, L., Eales, S., et al. 2011, MNRAS, 415, 64

de Jong, J. T. A., Verdoes Kleijn, G. A., Kuijken, K. H., \& Valentijn, E. A. 2012, ExA, 35, 25

Draine, B. T. 2009, in ASP Conf. Ser. 414, Cosmic Dust-Near and Far, ed. T.

Henning, E. Grün, \& J. Steinacker (San Francisco, CA: ASP), 453

Draine, B. T., Dale, D. A., Bendo, G., et al. 2007, ApJ, 663, 866

Driver, S. P., Hill, D. T., Kelvin, L. S., et al. 2011, MNRAS, 413, 971

Driver, S. P., Popescu, C. C., Tuffs, R. J., et al. 2007, MNRAS, 379, 1022

Driver, S. P., Robotham, A. S. G., Kelvin, L. S., et al. 2012, MNRAS, 427, 3244

Dunne, L., Gomez, H. L., da Cunha, E., et al. 2011, MNRAS, 417, 1510

Dwek, E. 1998, ApJ, 501, 643

Dwek, E., \& Cherchneff, I. 2011, ApJ, 727, 63

Dwek, E., \& Scalo, J. M. 1980, ApJ, 239, 193

Dwek, E., Staghun, J. G., \& Arendt, R. G. 2011, ApJ, 738, 36

Eales, S., Dunne, L., Clements, D., et al. 2010, PASP, 122, 499

Edmunds, M. G. 2001, MNRAS, 328, 223

Ferrarotti, A. S., \& Gail, H.-P. 2006, A\&A, 447, 553

Fritz, J., Gentile, G., Smith, M. W. L., et al. 2012, A\&A, 546, 34

Gail, H.-P., Zhukovska, S. V., Hoppe, P., \& Trieloff, M. 2009, ApJ, 698, 1136

Galliano, F., Dwek, E., \& Chanial, P. 2008, ApJ, 672, 214

Galliano, F., Madden, S. C., Jones, A. P., et al. 2003, A\&A, 407, 159

Galliano, F., Madden, S. C., Jones, A. P., Wilson, C. D., \& Bernard, J.-P. 2005, A\&A, 434, 867

Gomez, H. L., Clark, C. J. R., Nozawa, T., et al. 2011, MNRAS, 420, 3557

Gordon, K. D., Misselt, K. A., Witt, A. N., \& Clayton, G. C. 2001, ApJ, 551,269

Graham, A. W., \& Worley, C. C. 2008, MNRAS, 388, 1708

Green, D. A., Tuffs, R. J., \& Popescu, C. C. 2004, MNRAS, 355, 1315

Griffin, M. J., Abergel, A., Abreu, A., et al. 2010, A\&A, 518, L3

Häußler, B., Bamford, S. P., Vika, M., et al. 2013, MNRAS, 430, 330

Hill, D. T., Kelvin, L. S., Driver, S. P., et al. 2011, MNRAS, 412, 765

Ibar, E., Ivison, R. J., Cava, A., et al. 2010, MNRAS, 409, 38

Inoue, A. K. 2011, EP\&S, 63, 1027

James, A., Dunne, L., Eales, S., \& Edmunds, M. G. 2002, MNRAS, 335,753

Johnson, B. D., Schiminovich, D., Seibert, M., et al. 2007, ApJS, 173, 392

Jones, A. P., \& Nuth, J. A. 2011, A\&A, 530, A44

Jones, A. P., Tielens, A. G. G. M., \& Hollenbach, D. J. 1996, ApJ, 469, 740

Kelvin, L., Driver, S. P., Robotham, A. S. G., et al. 2012, MNRAS, 421, 1007

Kennicutt, R. C. 1998, ARA\&A, 36, 189

Kennicutt, R. C., Calzetti, D., Aniano., G., et al. 2011, PASP, 123, 1347
Kewley, L. J., \& Ellison, S. L. 2008, ApJ, 681, 1183

Kewley, L. J., Groves, B., Kaufmann, G., \& Heckmann, T. 2006, MNRAS, 372,961

Kong, X., Charlot, S., Brinchmann, J., \& Fall, S. M. 2004, MNRAS, 349, 769

Lakićević, M., Zanardo, G., van Loon, J. Th., et al. 2012, A\&A, 514, 2L

Laureijs, R., Amiaux, J., Arduini, S., et al. 2011, arXiv:1110.3193

Lintott, C. J., Schawinski, K., Slosar, A., et al. 2008, MNRAS, 389, 1179

MacLachlan, J. M., Matthews, L. D., Wood, K., \& Gallagher, J. S. 2011, ApJ, 741,6

Masters, K. L., Mosleh, M., Romer, A. K., et al. 2010, MNRAS, 404, 792

Matsuura, M., Dwek, E., Meixner, M., et al. 2011, Sci, 333, 1258

Mattsson, L., Anderson, A. C., \& Munkhammar, J. D. 2012, MNRAS, 423, 26

McKee, C. F. 1989, in IAU Symp. 135, Interstellar Dust, ed. L. J. Allamandola \& A. G. G. M. Tielens (Dordrecht: Kluwers), 431

Meurer, G. R., Heckmann, T. M., \& Calzetti, D. 1999, ApJ, 521, 64

Michałowski, M. J., Murphy, E. J., Hjorth, J., et al. 2010, A\&A, 522, A15

Misselt, K. A., Gordon, K. D., Clayton, G. C., \& Wolff, M. J. 2001, ApJ, 551,277

Molinari, S., Swinyard, B., Bally, J., et al. 2010, A\&A, 518L, 100

Möllenhoff, C., Popescu, C. C., \& Tuffs, R. J. 2006, A\&A, 456, 941

Morgan, H. L., \& Edmunds, M. G. 2003, MNRAS, 343, 427

Noll, S., Burgarella, D., Giovannoli, E., et al. 2009, A\&A, 507, 1793

Pascale, E., Auld, R., Dariush, A., et al. 2011, MNRAS, 415, 911

Pastrav, B., Popescu, C. C., Tuffs, R. J., \& Sansom, A. E. 2013, MNRAS, in press (arXiv: 1301.5602)

Peeples, M. S., \& Shankar, F. 2011, MNRAS, 417, 2962

Pierini, D., Gordon, K. D., Witt, A. N., \& Madsen, G. J. 2004, ApJ, 617, 1022

Pilbratt, G. L., Riedinger, J. R., Passvogel, T., et al. 2010, A\&A, 518L, 1

Poglitsch, A., Waelkens, C., Geis, N., et al. 2010, A\&A, 518L, 2

Popescu, C. C., Misiriotis, A., Kylafis, N. D., Tuffs, R. J., \& Fischera, J. 2000, A\&A, 362, 138

Popescu, C. C., Tuffs, R. J., Dopita, M. A., et al. 2011, A\&A, 527, A109

Rigby, E. E., Maddox, S. J., Dunne, L., et al. 2010, MNRAS, 415, 2336

Robotham, A., Driver, S. P., Norberg, P., et al. 2010, PASA, 27, 76

Rowlands, K., Dunne, L., Maddox, S., et al. 2011, MNRAS, 419, 2545

Salpeter, E. E. 1955, ApJ, 121, 161

Seibert, M., Martin, D. C., Heckman, T. M., et al. 2005, ApJL, 619, L55

Serra, P., Amblard, A., Temi, P., et al. 2011, ApJ, 740, 22

Silva, L., Granato, G. L., Bressan, A., \& Danese, L. 1998, ApJ, 509, 103

Skibba, R. A., Engelbracht, C. W., Dale, D., et al. 2011, ApJ, 738, 89

Smith, D. J. B., Dunne, L., Maddox, S. J., et al. 2011, MNRAS, 416,857

Smith, M. W. L., Gomez, H. L., Eales, S. A., et al. 2012, ApJ, 756, 40

Spergel, D. N., Verde, L., Peiris, H. V., et al. 2003, ApJS, 148, 175

Taylor, E. N., Hopkins, A. M., Baldry, I. K., et al. 2011, MNRAS, 418, 1587

Tremonti, C. A., Heckman, T. M., Kauffmann, G., et al. 2004, ApJ, 613, 898

Treyer, M., Shiminovich, D., Johnson, B., et al. 2007, ApJS, 173, 256

Tuffs, R. J., Drury, L. O'C., Fischera, J., et al. 1997, in Proc. First ISO Workshop on Analytical Spectroscopy, ed. A. M. Heras, K. Leech, N. R. Trams, \& Michael Perry (ESA SP-419; The Netherlands: Noordwijk), 177

Tuffs, R. J., Popescu, C. C., Völk, H. J., Kylafis, N. D., \& Dopita, M. A. 2004, A\&A, 419, 821T

Tully, R. B., \& Fisher, J. R. 1977, A\&A, 54, 661

Valiante, R., Schneider, R., Salvadori, S., \& Bianchi, S. 2011, MNRAS, 416, 1916

Weingartner, J. C., \& Draine, B. T. 2001, ApJ, 548, 296

Wijesinghe, D. B., Hopkins, A. M., Sharp, R., et al. 2011, MNRAS, 410, 2291

Xilouris, E. M., Byun, Y. I., Kylafis, N. D., Paleologou, E. V., \& Papamastorakis, J. 1999, A\&A, 344, 868

Zhukovska, S., Gail, H.-P., \& Trieloff, M. 2008, A\&A, 479, 453 